\title{
THE SURFACE DIFFUSION FLOW WITH ELASTICITY IN THE PLANE
}

\author{
NICOLA FUSCO, VESA JULIN, AND MASSIMILIANO MORINI
}

\begin{abstract}
In this paper we prove short-time existence of a smooth solution in the plane to the surface diffusion equation with an elastic term and without an additional curvature regularization. We also prove the asymptotic stability of strictly stable stationary sets.
\end{abstract}

\section{Contents}

1. Introduction

2. Preliminary results

2.1. Geometric preliminaries and notation

2.2. The energy functional

3. Short-time existence and regularity

4. Asymptotic Stability

5. Periodic graphs

6. Appendix

References

\section{INTRODUCTION}

In the last years, the physical literature has shown a rapidly growing interest toward the study of the morphological instabilities of interfaces between elastic phases generated by the competition between elastic and surface energies, the so called stress driven rearrangement instabilities (SDRI). They occur, for instance, in hetero-epitaxial growth of elastic films when a lattice mismatch between film and substrate is present, or in certain alloys that, under specific temperature conditions, undergo a phase separation in many small connected phases (that we call particles) within a common elastic body. A third interesting situation is represented by the formation and evolution of material voids inside a stressed elastic solid. Mathematically, the common thread is that equilibria are identified with local or global minimizers under a volume constraint of a free energy functional, which is given by the sum of the stored elastic energy and the surface energy (of isotropic or anisotropic perimeter type) accounting for the surface tension along the unknown profile of the film or the interface between the phases. The associated variational problems can be seen as non-local instances of the isoperimetric principle, where the non-locality is given by the elastic term. They are very well studied in the physical and numerical literature, but the available rigorous mathematical results are very few. We refer to $6,6,10,21,25,27$ for some existence, regularity and stability results related to a variational model describing the equilibrium configurations of two-dimensional epitaxially strained elastic films, and to [9, 15] for results in three-dimensions. We also mention that a hierarchy of variational principles to describe the equilibrium shapes in the aforementioned contexts has been introduced in [30. The simplest prototypical example is perhaps given 
by the following problem, which can be used to describe the equilibrium shapes of voids in elastically stressed solids (see for instance [36]):

$$
\operatorname{minimize} J(F):=\frac{1}{2} \int_{\Omega \backslash F} \mathbb{C} E\left(u_{F}\right): E\left(u_{F}\right) d z+\int_{\partial F} \varphi\left(\nu_{F}\right) d \sigma
$$

where minimization takes place among all sets $F \subset \Omega$ with prescribed measure $|F|=m$. Here, the set $F$ represents the shape of the void that has formed within the elastic body $\Omega$ (an open subset of $\mathbb{R}^{2}$ or $\mathbb{R}^{3}$ ), $u_{F}$ stands for the equilibrium elastic displacement in $\Omega \backslash F$ subject to a prescribed boundary conditions $u_{F}=w_{0}$ on $\partial \Omega$ (see (2.10) below), $\mathbb{C}$ is the elasticity tensor of the (linearly) elastic material, $E\left(u_{F}\right):=\left(\nabla u_{F}+\nabla^{T} u_{F}\right) / 2$ denotes the elastic strain of $u_{F}$, and $\varphi\left(\nu_{F}\right)$ is the anisotropic surface energy density evaluated at the outer unit normal $\nu_{F}$ to $F$. The presence of a nontrivial Dirichlet boundary condition $u_{F}=w_{0}$ on $\partial \Omega$ is what causes the solid $\Omega \backslash F$ to be elastically stressed. Indeed, note that when $w_{0}=0$ the elastic term becomes irrelevant and (1.1) reduces to the classical Wulff shape problem (with the confinement constraint $F \subseteq \Omega$ ). We refer to [14, 24] for related existence, regularity and stability results in two dimensions. See also [11] for a relaxation result valid in all dimensions for a variant of (1.1).

In this paper we address the evolutive counterpart of (1.1) in two-dimensions, namely the morphologic evolution of shapes towards equilbria of the functional $J$, driven by stress and surface diffusion. Assuming that mass transport in the bulk occurs at a much faster time scale, see [34], we have, according to the Einstein-Nernst relation, that the evolution is governed by the area preserving evolution law

$$
V_{t}=\partial_{\sigma \sigma} \mu_{t} \quad \text { on } \partial F(t)
$$

where $V_{t}$ denotes the (outer) normal velocity of the evolving curve $\partial F(t)$ at time $t$ and $\partial_{\sigma \sigma} \mu_{t}$ stands for the tangential laplacian of the chemical potential $\mu_{t}$ on $\partial F(t)$. In turn, $\mu_{t}$ is given by the first variation of the free-energy functional $J$ at $F(t)$, and thus (see (2.12) below) (1.2) takes the form

$$
V_{t}=\partial_{\sigma \sigma}\left(k_{\varphi, t}-Q\left(E\left(u_{F(t)}\right)\right)\right),
$$

where $k_{\varphi, t}$ is the anisotropic curvature of $\partial F(t), u_{F(t)}$ denotes as before the elastic equilibrium in $\Omega \backslash F(t)$ subject to $u_{F(t)}=w_{0}$ on $\partial \Omega$, and $Q$ is the quadratic form defined as $Q(A):=$ $\frac{1}{2} \mathbb{C} A: A$ for all $2 \times 2$-symmetric matrices $A$. Note that when $w_{0}=0$ the elastic term vanishes and thus (1.2) reduces to the surface diffusion flow equation

$$
V_{t}=\partial_{\sigma \sigma} k_{\varphi, t}
$$

for evolving curves, studied in [19] in the isotropic case (see also [20] for the $N$-dimensional case). Thus, we may also regard (1.3) as a sort of prototypical nonlocal perturbation of (1.4) by an additive elastic contribution.

As observed by Cahn and Taylor in the case without elasticity (see [13), the evolution equation (1.3) can be seen as the gradient flow of the energy functional $J$ with respect to a suitable Riemannian structure of $H^{-1}$ type, see Remark 3.1

When the anisotropy $\varphi$ is strongly elliptic, that is when it satisfies

$$
D^{2} \varphi(\nu) \tau \cdot \tau>0 \quad \text { for all } \nu \in \mathbb{S}^{1} \text { and all } \tau \perp \nu, \tau \neq 0
$$

the evolution (1.3) yields a parabolic 4-th order (geometric) equation, time by time coupled with the elliptic system describing the elastic equilibrium in $\Omega \backslash F(t)$. 
However, we mention here that for some physically relevant anisotropies the ellipticity condition (1.5) may fail at some directions $\nu$, see for instance [18, 36]. Whenever this happens, (1.3) becomes backward parabolic and thus ill-posed. To overcome this ill-posedness, a canonical approach inspired by Herring's work 31 consists in considering a regularized curvature-dependent surface energy of the form

$$
\int_{\partial F}\left(\varphi\left(\nu_{F}\right)+\frac{\varepsilon}{2} k^{2}\right) d \mathcal{H}^{1}
$$

where $\varepsilon>0$ and $k$ denotes the standard curvature, see [18, 29]. In this case (1.2) yields the following 6 -th order area preserving evolution equation

$$
V_{t}=\partial_{\sigma \sigma}\left(k_{\varphi, t}-Q\left(E\left(u_{F(t)}\right)\right)-\varepsilon\left(\partial_{\sigma \sigma} k+\frac{1}{2} k^{3}\right)\right) .
$$

This equation was studied numerically in [36] (see also [35, 12] and references therein) and analytically in 22, where local-in-time existence of a solution was established in the context of periodic graphs, modelling the evolution of epitaxially strained elastic films. We refer also to 23 for corresponding results in three-dimensions. We remark that the analysis of 22] (and of [23]), which is based on the so-called minimizing movements approach, relies heavily on the presence of the curvature regularization and, in fact, all the estimates provided there are $\varepsilon$-dependent and degenerate as $\varepsilon \rightarrow 0^{+}$, even when $\varphi$ is strongly elliptic. Thus, the methods developed in [22, 23] do not apply to the case $\varepsilon=0$ in (1.6).

In this paper we are able to address the case $\varepsilon=0$ and in one of the main results (see Theorems 3.2 and 3.8 below) we prove short time existence and uniqueness of a smooth solution of (1.3) starting from sufficiently regular initial sets. To the best of our knowledge this is the first existence result for the surface diffusion flow with elasticity and without curvature regularization. Note that in general one cannot expect global-in-time existence. Indeed, even when no elasticity is present and $\varphi$ is isotropic, singularities such as pinching may develop in finite time, see for instance [26].

In the second main result of the paper we establish global-in-time existence and study the long-time behavior for a class of initial data: we show that strictly stable stationary sets, that is, sets $E$ that are stationary for the energy functional $J$ and with positive second variation $\partial^{2} J(E)$ are exponentially stable for the flow (1.3). More precisely, if the initial set $F_{0}$ is sufficiently close to the strictly stable set $E$ and has the same area, then the flow (1.3) starting from $F_{0}$ exists for all times and converges to $E$ exponentially fast as $t \rightarrow+\infty$ (see Theorem 4.1 for the precise statement).

A few comments on the strategy of the proofs are in order. The main technical difficulties in proving short-time existence clearly originate from the presence of the nonlocal elastic term $Q\left(E\left(u_{F(t)}\right)\right)$ in (1.3). When a curvature regularization as in (1.6) is present, the elastic term may be regarded and treated as a lower order perturbation and thus is more easily handled. When $\varepsilon=0$ this is no longer possible and so one has to find a way to show that the parabolicity of the geometric part of the equation still tames the elastic contribution. Our strategy is based on the natural idea of thinking of $Q$ as a forcing term in order to set up a fixed point argument. Roughly speaking, given an initial set $F_{0}$ and a forcing term $f$, we let $t \mapsto F(t)$ be the flow starting from $F_{0}$ and solving

$$
V_{t}=\partial_{\sigma \sigma}\left(k_{\varphi, t}-f\right)
$$

and we consider the correspondig $t \mapsto Q\left(E\left(u_{F(t)}\right)\right)$, with $u_{F(t)}$ being as usual the elastic equilibrium in $\Omega \backslash F(t)$. The existence proof then amounts to finding a fixed point for the 
map $f \mapsto Q\left(E\left(u_{F(\cdot)}\right)\right)$. In order to implement this strategy, the crucial idea is to look at the squared $L^{2}$-norm of the tangential gradient of the chemical potential $\left(k_{\varphi, t}-f\right)$, that is, to study the behavior of the quantity

$$
\int_{\partial F(t)}\left(\partial_{\sigma}\left(k_{\varphi, t}-f\right)\right)^{2} d \mathcal{H}^{1}
$$

with respect to time. More precisely, by computing the time derivative of (1.7) we derive suitable energy inequalities involving (1.7) (see Lemma 3.3) yielding the a priori regularity estimates needed to carry out the fixed point argument. The quantity (1.7), with $f$ now given by the elastic term $Q$, is also crucial in the aforementioned asymptotic stability analysis. Here, by adapting to the present situation the methods developed in [1] for the surface diffusion flow without elasticity, we are able to show that for properly chosen initial sets, (1.7) becomes monotone decreasing in time and, in fact, exponentially decays to zero, thus giving the desired exponential convergence result.

This paper is organized as follows. In Section 2 we set up the problem, introduce the main notations and collect several auxiliary results concerning the energy functional $J$ in (1.1). Some of these results, which deal with the properties of strictly stable stationary sets, are then crucial for the asymptotic stability analysis carried out in Section 4. The short-time existence, uniqueness and regularity of the flow (1.3) for sufficiently regular initial data is addressed in Section 3 . In Section 5 we briefly illustrate how to apply our main existence and asymptotic stability results in the case of evolving periodic graphs, that is in the geometric setting considered in [22. In particular, in Theorem 5.1] we address and analytically characterize the exponential asymptotic stability of flat configurations, thus extending to the evolutionary setting the results of [25, 8]. In the final Appendix, for the reader's convenience we provide the proof of an interpolation result, probably known to the experts, that is used throughout the paper.

We conclude this introduction by mentioning that it would be interesting to investigate whether under the assumption (1.5) the flows (1.6) studied in 22 converge to (1.3) as $\varepsilon \rightarrow 0^{+}$, perhaps using the methods developed in [7. This issue as well as the extension of the results of this paper to three-dimensions will be addressed in future investigations.

\section{Preliminary Results}

2.1. Geometric preliminaries and notation. Let $F \subset \mathbb{R}^{2}$ be a bounded open set of class $C^{2}$. We denote the unit outer normal to $F$ by $\nu_{F}$ and the tangent vector $\tau_{F}$. Throughout the paper we choose the orientation so that $\tau_{F}=\mathcal{R} \nu_{F}$, where $\mathcal{R}$ is the counterclockwise rotation by $\pi / 2$.

The differential of a vector field $X$ along $\partial F$ is denoted by $\partial_{\sigma} X$. We recall that

$$
\partial_{\sigma} \nu_{F}=k_{F} \tau_{F} \quad \text { and } \quad \partial_{\sigma} \tau_{F}=-k_{F} \nu_{F},
$$

where $k_{F}$ is the curvature of $\partial F$. When no confusion arises, we will simply write $\nu, \tau$, and $k$ in place of $\nu_{F}, \tau_{F}$ and $k_{F}$. The tangential divergence of $X$ is $\operatorname{div}_{\tau} X:=\partial_{\sigma} X \cdot \tau$. The divergence theorem on $\partial F$ states that for every vector field $X \in C^{1}\left(\partial F ; \mathbb{R}^{2}\right)$ it holds

$$
\int_{\partial F} \operatorname{div}_{\tau} X d \mathcal{H}^{1}=\int_{\partial F} k X \cdot \nu d \mathcal{H}^{1}
$$


If the boundary of $F$ is of class $C^{m}$, with $m \geq 2$, then the signed distance function $d_{F}$ is of class $C^{m}$ in a tubular neighborhood of $\partial F$. We may extend $\nu, \tau$ and $k$ to such a neighborhood of $\partial F$ by setting $\nu:=\nabla d_{F}, \tau:=\mathcal{R} \nu$ and $k:=\operatorname{div} \nu=\Delta d_{F}$.

Throughout the paper, we fix a bounded Lipschitz open set $\Omega \subset \mathbb{R}^{2}$. Moreover, $G$ will always denote a smooth reference set, with the property that $G \subset \subset \Omega$. We will also denote by $\pi_{G}$ the orthogonal projection on $\partial G$ and by $\bar{\eta}$ a positive number such that

$$
d_{G} \text { and } \pi_{G} \text { are smooth in } \mathcal{N}_{\bar{\eta}}(\partial G),
$$

where $\mathcal{N}_{\bar{\eta}}(\partial G)$ denotes the $\bar{\eta}$-tubular neighborhood of $\partial G$.

We now introduce a class of sets $F$ sufficiently "close" to $G$ so that the boundary can be written as

$$
\partial F=\left\{x+h_{F}(x) \nu_{G}(x) \mid x \in \partial G\right\},
$$

for a suitable function $h_{F}$ defined on $\partial G$. More precisely, for $k \in \mathbb{N}$ and $\alpha \in(0,1)$ we set

$$
\begin{array}{r}
\mathfrak{h}_{M}^{k, \alpha}(G):=\left\{F \subset \subset \Omega:(2.3) \text { holds for some } h_{F} \in C^{k, \alpha}(\partial G),\right. \\
\text { with } \left.\left\|h_{F}\right\|_{L^{\infty}} \leq \bar{\eta} / 2 \text { and }\left\|h_{F}\right\|_{C^{k, \alpha}} \leq M\right\} .
\end{array}
$$

For such sets $F$ we also denote by $\pi_{F}^{-1}: \partial G \rightarrow \partial F$ the map $\pi_{F}^{-1}(x)=x+h_{F}(x) \nu_{G}(x)$ and set

$$
J_{F}:=\sqrt{\left(1+h_{F} k_{G}\right)^{2}+\left(\partial_{\sigma} h_{F}\right)^{2}},
$$

that is the tangential Jacobian on $\partial G$ of the map $\pi_{F}^{-1}$. We recall now some useful transformation formulas:

$$
\tau_{F} \circ \pi_{F}^{-1}=\frac{\left(1+h_{F} k_{G}\right) \tau_{G}+\partial_{\sigma} h_{F} \nu_{G}}{J_{F}}
$$

and

$$
\nu_{F} \circ \pi_{F}^{-1}=\frac{-\partial_{\sigma} h_{F} \tau_{G}+\left(1+h_{F} k_{G}\right) \nu_{G}}{J_{F}} .
$$

Similarly, the curvature $k_{F}$ of $F$ at $y=\pi_{F}^{-1}(x)$ is given by

$$
k_{F} \circ \pi_{F}^{-1}=\frac{-\partial_{\sigma \sigma} h_{F}\left(1+h_{F} k_{G}\right)+2\left(\partial_{\sigma} h_{F}\right)^{2} k_{G}+\left(1+h_{F} k_{G}\right)^{2} k_{G}+h_{F} \partial_{\sigma} h_{F} \partial_{\sigma} k_{G}}{J_{F}^{3}} .
$$

We now fix some notation, which will be used throughout the paper. If $t \mapsto F_{t}$ is a (smooth) flow of sets, in order to simplify the notation, we will sometimes write $h_{t}, \nu_{t}, \tau_{t}$, and $k_{t}$ instead of $h_{F_{t}}, \nu_{F_{t}}, \tau_{F_{t}}$, and $k_{F_{t}}$, respectively. Similarly, we will set $k_{\varphi, t}:=g\left(\nu_{t}\right) k_{t}$.

Moreover, whenever we have a one-parameter family $\left(g_{t}\right)_{t}$ of functions (or vector fields) we shall denote by $\dot{g}_{t}$ the partial derivative with respect to $t$ of the function $(x, t) \mapsto g_{t}(x)$, and by $\nabla^{k} g_{t}$ the $k$-th order differential of the function $(x, t) \mapsto g_{t}(x)$ with respect to $x$.

2.2. The energy functional. In this section we introduce the energy functional that underlies the flow. We also introduce the proper notions of stationary points and stability that will be needed in the study of the long-time behavior of the flow, see Section 4 .

As explained in the introduction, the free energy functional is the sum of an anisotropic perimeter and a bulk elastic term.

We start by introducing the anisotropic surface energy density, which is given by a positively one-homogeneous function $\varphi \in C^{\infty}\left(\mathbb{R}^{2} \backslash\{0\} ;(0,+\infty)\right)$ 


$$
D^{2} \varphi(\nu) \tau \cdot \tau \geq c_{0}>0
$$

for every $\nu \in \mathbb{S}^{1}$ and every $\tau \in \mathbb{S}^{1}$ such that $\tau \perp \nu$. Note that the above condition is equivalent to requiring that the level sets of $\varphi$ have positive curvature.

Concerning the elastic part, for $F \subset \subset \Omega$ and for the elastic displacement $u: \Omega \backslash F \rightarrow \mathbb{R}^{2}$ we denote by $E(u)$ the symmetric part of $\nabla u$, that is, $E(u):=\frac{\nabla u+(\nabla u)^{T}}{2}$. In what follows, $\mathbb{C}$ stands for a fourth order elasticity tensor acting on $2 \times 2$ symmetric matrices $A$, such that $\mathbb{C} A: A>0$ if $A \neq 0$. Finally we shall denote by $Q(A):=\frac{1}{2} \mathbb{C} A: A$ the elastic energy density.

We are now ready to write the energy functional. For a fixed boundary displacement $w_{0} \in H^{\frac{1}{2}}(\partial \Omega)$, we set

$$
J(F):=\int_{\partial F} \varphi\left(\nu_{F}\right) d \mathcal{H}^{1}+\int_{\Omega \backslash F} Q\left(E\left(u_{F}\right)\right) d x,
$$

where $u_{F}$ is the elastic equilibrium satisfying the Dirichlet boundary condition $w_{0}$ on a fixed relatively open subset $\partial_{D} \Omega \subseteq \partial \Omega$. More precisely, $u_{F}$ is the unique solution in $H^{1}\left(\Omega \backslash F ; \mathbb{R}^{2}\right)$ of the following elliptic system

$$
\begin{cases}\operatorname{div} \mathbb{C} E\left(u_{F}\right)=0 & \text { in } \Omega \backslash F, \\ \mathbb{C} E\left(u_{F}\right)\left[\nu_{F}\right]=0 & \text { on } \partial F \cup\left(\partial \Omega \backslash \partial_{D} \Omega\right), \\ u_{F}=w_{0} & \text { on } \partial_{D} \Omega .\end{cases}
$$

Next, we provide the first and the second variation formulas for (2.9). We start by recalling the well-known first variation formula for the anisotropic perimeter. To this aim, for any vector field $X \in C_{c}^{1}\left(\mathbb{R}^{2} ; \mathbb{R}^{2}\right)$, let $\left(\Phi_{t}\right)_{t \in(-1,1)}$ be the associated flow, that is the solution of

$$
\left\{\begin{array}{l}
\frac{\partial \Phi_{t}}{\partial t}=X\left(\Phi_{t}\right) \\
\Phi_{0}=I d
\end{array}\right.
$$

Then we have

$$
\left.\frac{d}{d t}\right|_{t=0} \int_{\partial \Phi_{t}(F)} \varphi\left(\nu_{\Phi_{t}(F)}\right) d \mathcal{H}^{1}=\int_{\partial F} k_{\varphi} X \cdot \nu d \mathcal{H}^{1},
$$

where the anisotropic curvature $k_{\varphi}$ of $\partial F$ is given by $k_{\varphi}:=\operatorname{div}_{\tau}(\nabla \varphi(\nu))$ and can be written also as

$$
\begin{aligned}
k_{\varphi} & =\operatorname{div}_{\tau}(\nabla \varphi(\nu))=\operatorname{div}(\nabla \varphi(\nu))=D^{2} \varphi(\nu): D \nu \\
& =\left(D^{2} \varphi(\nu) \tau \cdot \tau\right) k \\
& =: g(\nu) k
\end{aligned}
$$

on $\partial F$.

Concerning the full functional $J$, we have the following theorem.

Theorem 2.1. Let $F \subset \subset \Omega$ be a smooth set, $X \in C_{c}^{1}\left(\Omega ; \mathbb{R}^{2}\right)$ and let $\left(\Phi_{t}\right)_{t \in(-1,1)}$ be the associated flow as in (2.11). Set $\psi:=X \cdot \nu_{F}$ and $X_{\tau}:=\left(X \cdot \tau_{F}\right) \tau_{F}$ on $\partial F$. Then,

$$
\frac{d}{d t} J\left(\Phi_{t}(F)\right)_{t=0}=\int_{\partial F}\left(g\left(\nu_{F}\right) k_{F}-Q\left(E\left(u_{F}\right)\right)\right) \psi d \mathcal{H}^{1} .
$$


If in addition $\operatorname{div} X=0$ in a neighborhood of $\partial F$ we have

$$
\begin{aligned}
\frac{d^{2}}{d t^{2}} J\left(\left.\Phi_{t}(F)\right|_{t=0}\right. & =\int_{\partial F}\left[g\left(\nu_{F}\right)\left(\partial_{\sigma} \psi\right)^{2}-g\left(\nu_{F}\right) k_{F}^{2} \psi^{2}\right] d \mathcal{H}^{1}-2 \int_{\Omega \backslash F} Q\left(E\left(u_{\psi}\right)\right) d x \\
& -\int_{\partial F} \partial_{\nu_{F}}\left(Q\left(E\left(u_{F}\right)\right)\right) \psi^{2} d \mathcal{H}^{1}-\int_{\partial F}\left(g\left(\nu_{F}\right) k_{F}-Q\left(E\left(u_{F}\right)\right)\right) \operatorname{div}_{\tau}\left(\psi X_{\tau}\right) d \mathcal{H}^{1},
\end{aligned}
$$

where the function $u_{\psi}$ is the unique solution in $H^{1}\left(\Omega \backslash F ; \mathbb{R}^{2}\right)$, with $u_{\psi}=0$ on $\partial_{D} \Omega$, of

$$
\int_{\Omega \backslash F} \mathbb{C} E\left(u_{\psi}\right): E(\varphi) d x=-\int_{\partial F} \operatorname{div}_{\tau}\left(\psi E\left(u_{F}\right)\right) \cdot \varphi d \mathcal{H}^{1}
$$

for all $\varphi \in H^{1}\left(\Omega \backslash F ; \mathbb{R}^{2}\right)$ such that $\varphi=0$ on $\partial_{D} \Omega$.

Formulas (2.12) and (2.13) have been derived in 14 for the case where $\phi$ is the Euclidean norm, and in a slightly different setting, namely when $F$ is the subgraph of a periodic function, in [25, 9. The very same calculations apply to the more general situation considered here.

Throughout the paper, given a (sufficiently smooth) set $F \subset \subset \Omega$, we denote by $\Gamma_{F, 1}, \ldots, \Gamma_{F, m}$ the $m$ connected components of $\partial F$ and by $F_{i}$ the bounded open set enclosed by $\Gamma_{F, i}$. Note that the $F_{i}$ 's are not in general the connected components of $F$ and it may happen that $F_{i} \subset F_{j}$ for some $i \neq j$.

We are interested in area preserving variations, in the following sense.

Definition 2.2. Let $F \subset \subset \Omega$ be a smooth set. Given a vector field $X \in C_{c}^{\infty}\left(\Omega ; \mathbb{R}^{2}\right)$, we say that the associated flow $\left(\Phi_{t}\right)_{t \in(-1,1)}$ is admissible for $F$ if there exists $\varepsilon_{0} \in(0,1)$ such that

$$
\left|\Phi_{t}\left(F_{i}\right)\right|=\left|F_{i}\right| \quad \text { for } t \in\left(-\varepsilon_{0}, \varepsilon_{0}\right) \text { and } i=1, \ldots, m \text {. }
$$

Remark 2.3. Note that if the flow associated with $X$ is admissible in the sense of the previous definition, then for $i=1, \ldots, m$ we have

$$
\int_{\Gamma_{F, i}} X \cdot \nu_{F} d \mathcal{H}^{1}=0
$$

In view of this remark it is convenient to introduce the space $\tilde{H}^{1}(\partial F)$ consisting of all functions $\psi \in H^{1}(\partial F)$ with zero average on each component of $\partial F$, i.e.,

$$
\int_{\Gamma_{F, i}} \psi d \mathcal{H}^{1}=0 \quad \text { for every } i=1, \ldots, m .
$$

We observe that given $\psi \in \tilde{H}^{1}(\partial F) \cap C^{\infty}(\partial F)$ it is possible to construct a sequence of vector fields $X_{n} \in C_{c}^{\infty}\left(\Omega ; \mathbb{R}^{2}\right)$, with $\operatorname{div} X_{n}=0$ in a neighborhood of $\partial F$, such that $X_{n} \cdot \nu_{F} \rightarrow \psi$ in $C^{1}(\partial F)$, see [2, Proof of Corollary 3.4] for the details. Note that in particular the flows associated with $X_{n}$ are admissible.

Definition 2.4. Let $F \subset \subset \Omega$ be a set of class $C^{2}$. We say that $F$ is stationary if

$$
\frac{d}{d t} J\left(\left.\Phi_{t}(F)\right|_{t=0}=0\right.
$$

for all admissible flows in the sense of Definition 2.2 .

Remark 2.5. By Remark 2.3 and in view of (2.12) it follows that a set $F \subset \subset \Omega$ of class $C^{2}$ is stationary if and only if there exist constants $\lambda_{1}, \ldots, \lambda_{m}$ such that

$$
g\left(\nu_{F}\right) k_{F}-Q\left(E\left(u_{F}\right)\right)=\lambda_{i} \quad \text { on } \Gamma_{F, i}
$$


for every $i=1, \ldots, m$. In turn, note that if $F$ is stationary, then the second variation formula (2.13) reduces to

$$
\begin{aligned}
\frac{d^{2}}{d t^{2}} J\left(\left.\Phi_{t}(F)\right|_{t=0}=\right. & \int_{\partial F}\left[g\left(\nu_{F}\right)\left(\partial_{\sigma} \psi\right)^{2}-g\left(\nu_{F}\right) k_{F}^{2} \psi^{2}\right] d \mathcal{H}^{1} \\
& -2 \int_{\Omega \backslash F} Q\left(E\left(u_{\psi}\right)\right) d x-\int_{\partial F} \partial_{\nu_{F}}\left(Q\left(E\left(u_{F}\right)\right)\right) \psi^{2} d \mathcal{H}^{1},
\end{aligned}
$$

where we recall that $\psi=X \cdot \nu_{F}$ and $u_{\psi}$ is the function satisfying (2.14).

Note that if $F$ is a sufficiently regular (local) minimizer of (2.9) under the constraint $|F|=$ const., then there exists a constant $\lambda$ such that

$$
g\left(\nu_{F}\right) k_{F}-Q\left(E\left(u_{F}\right)\right)=\lambda \quad \text { on } \partial F .
$$

Thus, our notion of stationarity differs from the usual notion of criticality just recalled.

In view of (2.15), for any set $F \subset \subset \Omega$ of class $C^{2}$ it is convenient to introduce the quadratic form $\partial^{2} J(F)$ defined on $\tilde{H}^{1}(\partial F)$ as

$$
\begin{aligned}
\partial^{2} J(F)[\psi]:= & \int_{\partial F}\left[g\left(\nu_{F}\right)\left(\partial_{\sigma} \psi\right)^{2}-g\left(\nu_{F}\right) k_{F}^{2} \psi^{2}\right] d \mathcal{H}^{1} \\
& -2 \int_{\Omega \backslash F} Q\left(E\left(u_{\psi}\right)\right) d x-\int_{\partial F} \partial_{\nu_{F}}\left(Q\left(E\left(u_{F}\right)\right)\right) \psi^{2} d \mathcal{H}^{1},
\end{aligned}
$$

where $u_{\psi}$ is the unique solution of (2.14) under the Dirichlet condition $u_{\psi}=0$ on $\partial_{D} \Omega$. We conclude this section by defining the notion of stability for a stationary point.

Definition 2.6. Let $F \subset \subset \Omega$ be a stationary set in the sense of Definition 2.4. We say that $F$ is strictly stable if

$$
\partial^{2} J(F)[\psi]>0 \quad \text { for all } \psi \in \tilde{H}^{1}(\partial F) \backslash\{0\} .
$$

It is not difficult to see that (2.17) is equivalent to the coercivity of $\partial^{2} J(F)$ on $\tilde{H}^{1}(\partial F)$. More precisely, (2.17) holds if and only if there exists $m_{0}>0$ such that

$$
\partial^{2} J(F)[\psi] \geq m_{0}\|\psi\|_{\tilde{H}^{1}(\partial F)}^{2} \quad \text { for all } \psi \in \tilde{H}^{1}(\partial F),
$$

see [25]. In turn the latter coercivity property is stable with respect to small $C^{2, \alpha}$-perturbations. More precisely, we have:

Lemma 2.7. Assume that the reference set $G \subset \subset \Omega$ is a (smooth) strictly stable stationary set in the sense of Definition 2.6 and fix $\alpha \in(0,1)$. Then, there exists $\sigma_{0}>0$ such that for all $F \in \mathfrak{h}_{\sigma_{0}}^{2, \alpha}(G)$ (see (2.4) $)$ we have

$$
\partial^{2} J(F)[\psi] \geq \frac{m_{0}}{2}\|\psi\|_{\tilde{H}^{1}(\partial F)}^{2} \text { for all } \psi \in \tilde{H}^{1}(\partial F),
$$

where $m_{0}$ is the constant in (2.18).

Proof. The proof of the lemma goes as in [23, Proof of Lemma 4.12], where the case of $F$ being the subgraph of a periodic function of two variables is considered. Although the geometric framework here is more general, we can follow exactly the same line of argument up to the obvious changes due to the different setting (and some simplifications due the fact that here we work in two-dimensions). We refer the reader to the aforementioned reference for the details. 
Recall that $G_{1}, \ldots, G_{m}$ are the bounded open sets enclosed by the connected components $\Gamma_{G, 1}, \ldots, \Gamma_{G, m}$ of the boundary $\partial G$ of the reference set and observe that if $F \in \mathfrak{h}_{M}^{2, \alpha}(G)$, then $\partial F$ has the same number $m$ of connected components $\Gamma_{F, 1}, \ldots, \Gamma_{F, m}$, which can be numbered in such a way that

$$
\Gamma_{F, i}=\left\{x+h_{F}(x) \nu_{G}(x) \mid x \in \Gamma_{G, i}\right\}
$$

for suitable $h_{F} \in C^{k, \alpha}(\partial G)$.

In the next lemma we show that pairs of sets which are sufficiently close in a $C^{2, \alpha}$-sense can always be connected through area preserving flows in the sense of Definition 2.2. More precisely we have:

Lemma 2.8. Let $M>0$ and $\alpha \in(0,1)$. There exists $C>0$ with the following property: If $F_{1}, F_{2} \in \mathfrak{h}_{M}^{2, \alpha}(G)$ are such that $\left|F_{1, i}\right|=\left|F_{2, i}\right|, i=1, \ldots, m$, then there exists a flow $\left(\Phi_{t}\right)_{t \in(-1,1)}$ admissible for $F_{1}$ in the sense of Definition 2.2, such that $\Phi_{0}\left(F_{1}\right)=F_{1}, \Phi_{1}\left(F_{1}\right)=$ $F_{2},\left|\Phi_{t}\left(F_{1, i}\right)\right|=\left|F_{1, i}\right|$ for all $i=1, \ldots, m$ and $t \in[0,1]$. Moreover

$$
\sup _{t \in[0,1]}\left\|\Phi_{t}-I d\right\|_{C^{2, \alpha}\left(\mathcal{N}_{\bar{\eta} / 2}(\partial G)\right)} \leq C\left\|h_{F_{1}}-h_{F_{2}}\right\|_{C^{2, \alpha}(\partial G)}
$$

and the velocity field $X$ satisfies $\operatorname{div} X=0$ in the $\bar{\eta} / 2$-neighborhood $\mathcal{N}_{\bar{\eta} / 2}(\partial G)$. Here $F_{i, 1}, \ldots$, $F_{i, m}$ denote the bounded open sets enclosed by the connected components $\Gamma_{F_{i}, 1}, \ldots, \Gamma_{F_{i}, m}$ of $\partial F_{i}, i=1,2$, which are supposed to be numbered as in (2.19).

Proof. We adapt the construction of [33, Proposition 3.4]. We start by constructing a $C^{\infty}$ vector-field $\tilde{X}: \mathcal{N}_{\bar{\eta}}(\partial G) \rightarrow \mathbb{R}^{2}$ satisfying

$$
\operatorname{div} \tilde{X}=0 \quad \text { in } \mathcal{N}_{\bar{\eta}}(\partial G), \quad \tilde{X}=\nu_{G} \quad \text { on } \partial G .
$$

To this aim, let $\zeta$ be the solution of

$$
\begin{cases}\nabla \zeta \cdot \nabla d_{G}+\zeta \Delta d_{G}=0 & \text { in } \mathcal{N}_{\bar{\eta}}(\partial G) \\ \zeta=1 & \text { on } \partial G\end{cases}
$$

We may solve the above PDE by the method of characteristics, constructing such a $\zeta$ amounts to solving for every $x \in \partial G$ the Cauchy problem

$$
\left\{\begin{array}{l}
\left(f_{x}\right)^{\prime}(t)+f_{x}(t) \Delta d_{G}\left(x+t \nu_{G}(x)\right)=0 \quad \text { in }(-\bar{\eta}, \bar{\eta}) \\
f_{x}(0)=1
\end{array}\right.
$$

and setting

$$
\zeta\left(x+t \nu_{G}(x)\right):=f_{x}(t)=\exp \left(-\int_{0}^{t} \Delta d_{G}\left(x+s \nu_{G}(x)\right) d s\right) .
$$

We can now define $\tilde{X}:=\zeta \nabla d_{G}$ and check that $\operatorname{div}\left(\zeta \nabla d_{G}\right)=\nabla \zeta \cdot \nabla d_{G}+\zeta \Delta d_{G}=0$.

Let now $F_{1}$ and $F_{2}$ be as in the statement. We choose $X \in C_{c}^{\infty}\left(\Omega ; \mathbb{R}^{2}\right)$ in such a way that

$$
X(x):=\left(\int_{h_{F_{1}}\left(\pi_{G}(x)\right)}^{h_{F_{2}}\left(\pi_{G}(x)\right)} \frac{d s}{\zeta\left(\pi_{G}(x)+s \nu_{G}\left(\pi_{G}(x)\right)\right)}\right) \tilde{X}(x) \quad \text { for every } x \in \mathcal{N}_{\bar{\eta} / 2}(\partial G)
$$

and we let $\Phi$ be the associated flow. Notice that the integral appearing in (2.22) represents the time needed to go from $\pi_{G}(x)+h_{F_{1}}\left(\pi_{G}(x)\right) \nu_{G}\left(\pi_{G}(x)\right)$ to $\pi_{G}(x)+h_{F_{2}}\left(\pi_{G}(x)\right) \nu_{G}\left(\pi_{G}(x)\right)$ along the trajectory of the vector field $\tilde{X}$. Therefore the above definition ensures that the time needed to go from $\pi_{G}(x)+h_{F_{1}}\left(\pi_{G}(x)\right) \nu_{G}\left(\pi_{G}(x)\right)$ to $\pi_{G}(x)+h_{F_{2}}\left(\pi_{G}(x)\right) \nu_{G}\left(\pi_{G}(x)\right)$ along the modified vector field $X$ is one. This is equivalent to saying that for all $x \in \partial G$ we have 
$\Phi_{1}\left(x+h_{F_{1}}(x) \nu_{G}(x)\right)=x+h_{F_{2}}(x) \nu_{G}(x)$ and, in turn, $\Phi_{1}\left(F_{1}\right)=F_{2}$. Moreover, recalling the first equation in (2.21) and using the fact that the function

$$
x \mapsto \int_{h_{F_{1}}\left(\pi_{G}(x)\right)}^{h_{F_{2}}\left(\pi_{G}(x)\right)} \frac{d s}{\zeta\left(\pi_{G}(x)+s \nu_{G}\left(\pi_{G}(x)\right)\right)}
$$

is constant along the trajectories of $\tilde{X}$, we deduce from (2.22) that the modified field $X$ is divergence free in $\mathcal{N}_{\bar{\eta} / 2}(\partial G)$. Note that by (2.22) it also follows

$$
\|X\|_{C^{2, \alpha}\left(\mathcal{N}_{\bar{\eta} / 2}(\partial G)\right)} \leq C\left\|h_{F_{1}}-h_{F_{2}}\right\|_{C^{2, \alpha}(\partial G)}
$$

for a constant $C>0$ depending on $G$, and thus (2.20) easily follows.

Observe now that for $i=1, \ldots, m$ and for $\varepsilon_{0}>0$ small enough by [17, equation (2.30)] we have

$$
\frac{d^{2}}{d t^{2}}\left|\Phi_{t}\left(F_{1, i}\right)\right|=\int_{\Phi_{t}\left(\Gamma_{F_{i}, 1}\right)}(\operatorname{div} X)\left(X \cdot \nu_{\Phi_{t}\left(F_{1, i}\right)}\right)=0 \quad \text { for all } t \in\left[-\varepsilon_{0}, 1\right],
$$

where we used the fact that $X$ is divergence free in $\mathcal{N}_{\bar{\eta} / 2}(\partial G)$. Hence the function $t \mapsto$ $\left|\Phi_{t}\left(F_{1, i}\right)\right|$ is affine in $\left[-\varepsilon_{0}, 1\right]$. Since by assumption $\left|\Phi_{0}\left(F_{1, i}\right)\right|=\left|F_{1, i}\right|=\left|F_{2, i}\right|=\left|\Phi_{1}\left(F_{1, i}\right)\right|$, it is in fact constant. This concludes the proof of the lemma.

We conclude this section by showing that in a sufficiently small $C^{2, \alpha}$-neighborhood of $G$ the stationary sets are isolated, once we fix the areas enclosed by the connected components of the boundary.

Proposition 2.9. Assume that the reference set $G \subset \subset \Omega$ is a (smooth) strictly stable stationary set in the sense of Definition 2.6. fix $\alpha \in(0,1)$, and let $\sigma_{0}$ be the constant provided by Lemma 2.7. There exists $\sigma_{1} \in\left(0, \sigma_{0}\right)$ with the following property: Let $F_{1}, F_{2} \in \mathfrak{h}_{\sigma_{1}}^{2, \alpha}(G)$ be stationary sets in the sense of Definition 2.4 and (with same notation of Lemma 2.8) assume that $\left|F_{1, i}\right|=\left|F_{2, i}\right|$ for $i=1, \ldots, m$. Then $F_{1}=F_{2}$.

Proof. We start by observing that for any $\eta \in\left(0, \sigma_{0}\right)$ we may choose $\sigma_{1}>0$ so small that for any pair $F_{1}, F_{2} \in \mathfrak{h}_{\sigma_{1}}^{2, \alpha}(G)$ the flow $\Phi_{t}$ provided by Lemma 2.8 satisfies

$$
\Phi_{t}\left(F_{1}\right) \in \mathfrak{h}_{\eta}^{2, \alpha}(G) \subseteq \mathfrak{h}_{\sigma_{0}}^{2, \alpha}(G) \quad \text { for all } t \in[0,1],
$$

Notice that this is possible thanks to (2.20).

Recall that by Remark 2.5 there exist constants $\lambda_{i}$ such that $g\left(\nu_{G}\right) k_{G}-Q\left(E\left(u_{G}\right)\right)=\lambda_{i}$ on $\Gamma_{G, i}$ for $i=1, \ldots, m$. In what follows, the subscript $t$ stands for the subscript $\Phi_{t}\left(F_{1}\right)$, where $\Phi_{t}$ is the flow of Lemma 2.8. Fix $\varepsilon>0$ and observe that by taking $\eta$ in (2.23) and, in turn, $\sigma_{1}$ smaller if needed, we may ensure that

$$
\sup _{t \in[0,1]}\left\|\nu_{t}-\nu_{G} \circ \pi_{G}\right\|_{C^{1}\left(\Phi_{t}(\partial F)\right)} \leq \varepsilon
$$

and

$$
\sup _{i=1, \ldots, m} \sup _{t \in[0,1]}\left\|g\left(\nu_{t}\right) k_{t}-Q\left(E\left(u_{t}\right)\right)-\lambda_{i}\right\|_{C^{0}\left(\Phi_{t}\left(\Gamma_{F, i}\right)\right)} \leq \varepsilon .
$$

The latter condition can be guaranteed thanks also to the elliptic estimates proved later in Lemma 3.6. Let $X$ be the velocity field of $\Phi_{t}$ and recall that by the explicit construction given in the proof of Lemma 2.8 we have $X=\left[X \cdot\left(\nu_{G} \circ \pi_{G}\right)\right] \nu_{G} \circ \pi_{G}$ in $\mathcal{N}_{\bar{\eta} / 2}(\partial G)$. Thus, writing 
$X=\left[X \cdot\left(\nu_{G} \circ \pi_{G}-\nu_{t}\right)\right] \nu_{G} \circ \pi_{G}+\left(X \cdot \nu_{t}\right) \nu_{G} \circ \pi_{G}$ on $\Phi_{t}(\partial F)$ and using (2.24) with $\varepsilon$ (and in turn $\left.\sigma_{1}\right)$ sufficiently small, we find that for all $t \in[0,1]$

$$
\left.|X| \leq 2\left|X \cdot \nu_{t}\right| \quad \text { and } \quad\left|\partial_{\sigma} X\right| \leq C\left(\left|X \cdot \nu_{t}\right|+\left|\partial_{\sigma}\left(X \cdot \nu_{t}\right)\right|\right)\right) \quad \text { on } \Phi_{t}(\partial F),
$$

for some constant $C>0$ depending only on $G$.

Let now $F_{1}$ and $F_{2}$ be as in the statement of the proposition and $\Phi_{t}$ as above. Recalling (2.13) and (2.16), for every $s \in[0,1]$ we may write

$$
\begin{aligned}
\frac{d^{2}}{d t^{2}} J\left(\left.\Phi_{t}(F)\right|_{t=s}\right. & =\partial^{2} J\left(\Phi_{s}\left(F_{1}\right)\right)\left[X \cdot \nu_{s}\right] \\
& -\int_{\Phi_{s}\left(\partial F_{1}\right)}\left[g\left(\nu_{s}\right) k_{s}-Q\left(E\left(u_{s}\right)\right)\right] \operatorname{div}_{\tau}\left(X_{\tau}\left(X \cdot \nu_{s}\right)\right) d \mathcal{H}^{1} \\
& =\partial^{2} J\left(\Phi_{s}\left(F_{1}\right)\right)\left[X \cdot \nu_{s}\right] \\
& -\sum_{i=1}^{m} \int_{\Phi_{s}\left(\Gamma_{F_{1}, i}\right)}\left[g\left(\nu_{s}\right) k_{s}-Q\left(E\left(u_{s}\right)\right)-\lambda_{i}\right] \operatorname{div}_{\tau}\left(X_{\tau}\left(X \cdot \nu_{s}\right)\right) d \mathcal{H}^{1}
\end{aligned}
$$

Recall that $\left(\Phi_{t}\right)$ is an admissible flow and thus $X \cdot \nu_{s} \in \tilde{H}^{1}\left(\Phi_{s}\left(\partial F_{1}\right)\right)$ for every $s \in[0,1]$ due to Remark 2.3. In turn, by (2.23) and Lemma 2.7 we deduce that

$$
\partial^{2} J\left(\Phi_{s}\left(F_{1}\right)\right)\left[X \cdot \nu_{s}\right] \geq \frac{m_{0}}{2}\left\|X \cdot \nu_{s}\right\|_{\tilde{H}^{1}\left(\Phi_{s}\left(\partial F_{1}\right)\right)}^{2} .
$$

Note also that by (2.26) it is easily checked that

$$
\left\|\operatorname{div}_{\tau}\left(X_{\tau}\left(X \cdot \nu_{s}\right)\right)\right\|_{L^{1}\left(\Phi_{s}\left(F_{1}\right)\right)} \leq C\left\|X \cdot \nu_{s}\right\|_{\tilde{H}^{1}\left(\Phi_{s}\left(\partial F_{1}\right)\right)}^{2} .
$$

Thus, collecting all the above observations and recalling also (2.25), we deduce from (2.27) that for every $s \in[0,1]$

$$
\frac{d^{2}}{d t^{2}} J\left(\left.\Phi_{t}(F)\right|_{t=s} \geq\left(\frac{m_{0}}{2}-C m \varepsilon\right)\left\|X \cdot \nu_{s}\right\|_{\tilde{H}^{1}\left(\Phi_{s}\left(\partial F_{1}\right)\right)}^{2} \geq \frac{m_{0}}{4}\left\|X \cdot \nu_{s}\right\|_{\tilde{H}^{1}\left(\Phi_{s}\left(\partial F_{1}\right)\right)}^{2},\right.
$$

where the last inequality holds true provided we choose in (2.25) a sufficiently small $\varepsilon$ (and $\left.\sigma_{1}\right)$. Since on the other hand by the stationarity of $F_{1}$ and $F_{2}$ we have

$$
\frac{d}{d t} J\left(\left.\Phi_{t}(F)\right|_{t=0}=\frac{d}{d t} J\left(\left.\Phi_{t}(F)\right|_{t=1}=0\right.\right.
$$

we infer that $\frac{d^{2}}{d t^{2}} J\left(\left.\Phi_{t}(F)\right|_{t=s}=0\right.$ and in turn $X \cdot \nu_{s}=0$ on $\Phi_{s}\left(\partial F_{1}\right)$ for all $s \in[0,1]$. This means that $s \mapsto \Phi_{s}\left(F_{1}\right)$ is constant in $[0,1]$ and, in particular, $F_{1}=F_{2}$.

\section{Short-time EXISTENCE AND REgUlarity}

We are interested in the evolution law

$$
V_{t}=\partial_{\sigma \sigma}\left(g\left(\nu_{t}\right) k_{t}-Q\left(E\left(u_{t}\right)\right)\right) \quad \text { on } \partial F_{t},
$$

where $V_{t}$ stands for the outer normal velocity of $\partial F_{t}$, and $u_{t} \in H^{1}\left(\Omega \backslash F_{t} ; \mathbb{R}^{2}\right)$ is the unique solution of

$$
\begin{cases}\operatorname{div} \mathbb{C} E\left(u_{t}\right)=0 & \text { in } \Omega \backslash F_{t} \\ \mathbb{C} E\left(u_{t}\right)\left[\nu_{t}\right]=0 & \text { on } \partial F_{t} \cup\left(\partial \Omega \backslash \partial_{D} \Omega\right), \\ u_{t}=w_{0} & \text { on } \partial_{D} \Omega\end{cases}
$$


Remark 3.1. We remark that (3.1) can be regarded as the gradient flow of (2.9) with respect to a suitable Riemannian structure of $H^{-1}$-type. To illustrate this fact, consider the dual $H_{t}^{-1}$ of $H_{t}^{1}:=H^{1}(\partial F(t))$ endowed with the scalar product

$$
\begin{aligned}
\left\langle\psi_{1}, \psi_{2}\right\rangle_{H_{t}^{-1}}:=\int_{\partial F(t)} \partial_{\sigma} v_{\psi_{1}} \partial_{\sigma} v_{\psi_{2}} d \mathcal{H}^{1}=-\left\langle\partial_{\sigma \sigma} v_{\psi_{2}}, v_{\psi_{1}}\right\rangle_{H_{t}^{-1} \times H_{t}^{1}} & \\
& =\left\langle\psi_{2}, v_{\psi_{1}}\right\rangle_{H_{t}^{-1} \times H_{t}^{1}}=\left\langle\psi_{1}, v_{\psi_{2}}\right\rangle_{H_{t}^{-1} \times H_{t}^{1}}
\end{aligned}
$$

where $\partial_{\sigma}$ denotes the tangential derivative on $\partial F(t)$ and for any $\psi \in H_{t}^{-1}$ the function $v_{\psi}$ is the unique function in $H_{t}^{1}$ satisfying

$$
\left\{\begin{array}{l}
-\partial_{\sigma \sigma} v_{\psi}=\psi \\
\int_{\partial F(t)} v_{\psi} d \mathcal{H}^{1}=0 .
\end{array} \text { on } \partial F(t),\right.
$$

As already recalled, the first variation $\partial J(F(t))$ satisfies

$$
\partial J(F(t))[\psi]=\int_{\partial F(t)}\left(k_{\varphi, t}-Q\left(E\left(u_{F(t)}\right)\right)\right) \psi d \mathcal{H}^{1}
$$

for all $\psi \in C^{\infty}(\partial F(t))$. Thus, recalling also (3.1), (3.3) and (3.4), we have

$$
\begin{aligned}
\left\langle V_{t}, \psi\right\rangle_{H^{-1}(\partial F(t))} & =\int_{\partial F(t)} V_{t} v_{\psi} d \mathcal{H}^{1}=\int_{\partial F(t)} \partial_{\sigma \sigma}\left(k_{\varphi, t}-Q\left(E\left(u_{F(t)}\right)\right)\right) v_{\psi} d \mathcal{H}^{1} \\
& =\int_{\partial F(t)}\left(k_{\varphi, t}-Q\left(E\left(u_{F(t)}\right)\right)\right) \partial_{\sigma \sigma} v_{\psi} d \mathcal{H}^{1}=-\partial J(F(t))[\psi] .
\end{aligned}
$$

Hence, time by time the normal velocity $V_{t}$ is the element of $H_{t}^{-1}$ that represents the action of $-\partial J(F(t))$ with respect to the scalar product defined in (3.3). This formally establishes the $H^{-1}$-gradient flow structure of (3.1).

The following theorem establishes the short-time existence of a unique weak solution of (3.1). In Theorem 3.8 below we will show that the weak solution is in fact smooth and therefore classical.

Theorem 3.2. Let $F_{0} \subset \subset \Omega$ be such that

$$
\partial F_{0}=\left\{x+h_{0}(x) \nu_{G}(x) \mid h_{0} \in H^{3}(\partial G)\right\} .
$$

There exist $\delta$ and $T>0$, which depend on the $H^{3}$-norm of $h_{0}$, such that if $\left\|h_{0}\right\|_{L^{2}(\partial G)} \leq \delta$ then the flow (3.1) admits a unique local-in-time weak solution $\left(F_{t}\right)_{t \in(0, T)}$ with an initial set $F_{0}$. More precisely, we have $\partial F_{t}=\left\{x+h_{t}(x) \nu_{G}(x)\right\}$, where $\left(h_{t}\right)_{t} \in H^{1}\left(0, T ; H^{1}(\partial G)\right) \cap$ $L^{2}\left(0, T ; H^{3}(\partial G)\right)$. Moreover $\left(h_{t}\right)_{t} \in C^{0}\left([0, T) ; C^{2, \alpha}(\partial G)\right)$ for all $\alpha \in\left(0, \frac{1}{2}\right)$ and $\left(\left[g\left(\nu_{t}\right) k_{t}-\right.\right.$ $\left.\left.Q\left(E\left(u_{t}\right)\right)\right] \circ \pi_{F_{t}}^{-1}\right)_{t} \in L^{2}\left(0, T ; H^{3}(\partial G)\right)$.

Note that when the initial set $F_{0}$ is smooth we may take $G=F_{0}$. We give the proof of Theorem 3.2 at the end of the section. We will first prove a sequence of lemmas needed for the proof of the short-time existence result.

We will need some preliminary results. Our proof of Theorem 3.2 is based on a fixed point argument. To this aim, for a given smooth function $f: \partial G \times(0, T) \rightarrow \mathbb{R}$, we consider the forced surface diffusion flow given by

$$
V_{t}=\partial_{\sigma \sigma}\left(g\left(\nu_{t}\right) k_{t}+f_{t} \circ \pi_{G}\right) \quad \text { on } \partial F_{t}
$$


with initial datum $F_{0}$ of class $H^{3}$, where we denoted $f_{t}:=f(\cdot, t)$. To simplify the notation we will denote

$$
R_{t}:=g\left(\nu_{t}\right) k_{t}+f_{t} \circ \pi_{G} \quad \text { on } \partial F_{t} .
$$

The following monotone quantities are the starting point of our analysis.

Lemma 3.3. Let $F_{0}$ be a set with smooth boundary, $f \in C^{\infty}(\partial G \times[0, \infty))$, and let $\left(F_{t}\right)_{t \in(0, T)}$ be a smooth flow satisfying (3.6), with initial datum $F_{0}$. Then we have

$$
\frac{d}{d t} \int_{F_{t} \Delta G} \operatorname{dist}(x, \partial G) d x=\int_{\partial F_{t}} d_{G} \partial_{\sigma \sigma} R_{t} d \mathcal{H}^{1} \leq P\left(F_{t}\right)^{\frac{1}{2}}\left(\int_{\partial F_{t}}\left(\partial_{\sigma} R_{t}\right)^{2} d \mathcal{H}^{1}\right)^{\frac{1}{2}}
$$

whenever the flow (3.6) exists. Moreover there exists $C_{1}$, which depends on $\sup _{(0, T)}\left\|h_{t}\right\|_{C^{2, \alpha}}$ and $\sup _{(0, T)}|| f_{t} \|_{C^{1, \alpha},}$ such that

$$
\begin{aligned}
\frac{d}{d t} \int_{\partial F_{t}}\left(\partial_{\sigma} R_{t}\right)^{2} d \mathcal{H}^{1}+c_{0} \int_{\partial F_{t}}\left(\partial_{\sigma \sigma \sigma}\right. & \left.R_{t}\right)^{2} d \mathcal{H}^{1} \\
\leq & \leq C_{1}\left\|\dot{f}_{t}\right\|_{H^{-\frac{1}{2}}(\partial G)}^{2}+C_{1}\left(1+\int_{\partial F_{t}}\left(\partial_{\sigma} R_{t}\right)^{2} d \mathcal{H}^{1}\right)^{q}
\end{aligned}
$$

for some $q>1$.

Proof. Let $X_{t}$ be the velocity field associated with the flow. In particular we have that

$$
X_{t} \cdot \nu_{t}=\partial_{\sigma \sigma} R_{t} .
$$

For $t \in(0, T)$ and $s>0 \Phi_{s}: \partial F_{t} \rightarrow \partial F_{t+s}, \Phi_{s}=\pi_{F_{t+s}}^{-1} \circ \pi_{F_{t}}$ are admissible diffeomorphisms and by the above equality it holds $\left.\frac{\partial}{\partial s} \Phi_{s}\right|_{s=0}=X_{t}$.

As mentioned in the previous section we can extend $\nu_{t}, \tau_{t}$ and $k_{t}$ by means of the signed distance function $d_{F_{t}}$ in a tubular neighborhood of $\partial F_{t}$. This extension yields the following identities (see for instance [9, Lemma 4.2]):

$$
\begin{gathered}
\partial_{\nu_{t}} k_{\varphi, t}=-k_{t}^{2} g\left(\nu_{t}\right), \\
\dot{\nu}_{t}=-\partial_{\sigma}\left(X_{t} \cdot \nu_{t}\right) \tau_{t}=-\partial_{\sigma \sigma \sigma} R_{t} \tau_{t}
\end{gathered}
$$

and

$$
\dot{k}_{\varphi, t}=\operatorname{div}\left(D^{2} \varphi\left(\nu_{t}\right) \dot{\nu}_{t}\right)=-\partial_{\sigma}\left(g\left(\nu_{t}\right) \partial_{\sigma \sigma \sigma} R_{t}\right) .
$$

Note that

$$
\int_{F_{t} \Delta G} \operatorname{dist}(x, \partial G) d x=\int_{F_{t}} d_{G} d x-\int_{G} d_{G} d x
$$

Thus,

$$
\begin{aligned}
\frac{d}{d t} \int_{F_{t} \Delta G} \operatorname{dist}(x, \partial G) d x & =\frac{d}{d t} \int_{F_{t}} d_{G} d x=\int_{F_{t}} \operatorname{div}\left(d_{G} X_{t}\right) d x \\
& =\int_{\partial F_{t}} d_{G}\left(X_{t} \cdot \nu_{t}\right) d \mathcal{H}^{1}=\int_{\partial F_{t}} d_{G} \partial_{\sigma \sigma} R_{t} d \mathcal{H}^{1} \\
& =-\int_{\partial F_{t}} \partial_{\sigma} d_{G} \partial_{\sigma} R_{t} d \mathcal{H}^{1} \leq P\left(F_{t}\right)^{\frac{1}{2}}\left(\int_{\partial F_{t}}\left(\partial_{\sigma} R_{t}\right)^{2} d \mathcal{H}^{1}\right)^{\frac{1}{2}} .
\end{aligned}
$$


This proves (3.8). Concerning (3.9) we begin by calculating

$$
\begin{gathered}
\frac{d}{d t}\left(\frac{1}{2} \int_{\partial F_{t}}\left(\partial_{\sigma} R_{t}\right)^{2} d \mathcal{H}^{1}\right)=\left.\frac{\partial}{\partial s}\left(\frac{1}{2} \int_{\partial F_{t}}\left(\left(\nabla R_{t+s}\right)\left(\Phi_{s}(x)\right) \cdot \tau_{t+s}\left(\Phi_{s}(x)\right)\right)^{2} J_{\tau} \Phi_{s} d \mathcal{H}^{1}\right)\right|_{s=0} \\
\quad=\frac{1}{2} \int_{\partial F_{t}}\left(\partial_{\sigma} R_{t}\right)^{2} \operatorname{div}_{\tau} X_{t} d \mathcal{H}^{1}+\left.\int_{\partial F_{t}} \partial_{\sigma} R_{t} \frac{\partial}{\partial s}\left(\nabla R_{t+s}\left(\Phi_{s}(x)\right) \cdot \tau_{t+s}\left(\Phi_{s}(x)\right)\right)\right|_{s=0} d \mathcal{H}^{1} .
\end{gathered}
$$

Using our notation we write the last term as

$$
\begin{aligned}
& \left.\frac{\partial}{\partial s}\left(\nabla R_{t+s}\left(\Phi_{s}(x)\right) \cdot \tau_{t+s}\left(\Phi_{s}(x)\right)\right)\right|_{s=0} \\
& \quad=\partial_{\sigma} \dot{R}_{t}+\left(\nabla^{2} R_{t} X_{t}\right) \cdot \tau_{t}+\nabla R_{t} \cdot \dot{\tau_{t}}+\nabla R_{t} \cdot\left(\nabla \tau_{t} X_{t}\right) .
\end{aligned}
$$

We write $X_{t, \tau}:=X_{t} \cdot \tau_{t}$. Note that by (3.11) we have that $\dot{\tau}_{t}=\mathcal{R} \dot{\nu}_{t}=\partial_{\sigma \sigma \sigma} R_{t} \nu_{t}$. Moreover it holds $D \tau_{t} \nu_{t}=0$. Therefore we get

$$
\begin{aligned}
& \left.\frac{\partial}{\partial s}\left(\nabla R_{t+s}\left(\Phi_{s}(x)\right) \cdot \tau_{t+s}\left(\Phi_{s}(x)\right)\right)\right|_{s=0} \\
& \quad=\partial_{\sigma} \dot{R}_{t}+\partial_{\sigma \sigma \sigma} R_{t} \partial_{\nu_{t}} R_{t}+\partial_{\sigma \sigma} R_{t}\left(\nabla^{2} R_{t} \nu_{t}\right) \cdot \tau_{t}+\left(\nabla^{2} R_{t} \tau_{t}\right) \cdot \tau_{t} X_{t, \tau}+\nabla R_{t} \cdot\left(\nabla \tau_{t} \tau_{t}\right) X_{t, \tau}
\end{aligned}
$$

Therefore, using the fact that $\partial_{\sigma}\left(\partial_{\nu_{t}} R_{t}\right)=k_{t} \partial_{\sigma} R_{t}+\left(\nabla^{2} R_{t} \nu_{t}\right) \cdot \tau_{t}$ and integrating by parts, (3.13) can be written as

$$
\begin{aligned}
\frac{d}{d t} & \left(\frac{1}{2} \int_{\partial F_{t}}\left(\partial_{\sigma} R_{t}\right)^{2} d \mathcal{H}^{1}\right)=\int_{\partial F_{t}} \frac{1}{2}\left(\partial_{\sigma} R_{t}\right)^{2} \operatorname{div}_{\tau} X_{t}+\partial_{\sigma} R_{t} \partial_{\sigma} \dot{R}_{t}+\partial_{\sigma} R_{t} \partial_{\sigma \sigma \sigma} R_{t} \partial_{\nu_{t}} R_{t} d \mathcal{H}^{1} \\
& +\int_{\partial F_{t}} \partial_{\sigma} R_{t} \partial_{\sigma \sigma} R_{t}\left(\nabla^{2} R_{t} \nu_{t}\right) \cdot \tau_{t}+\partial_{\sigma} R_{t} X_{t, \tau}\left(\nabla^{2} R_{t} \tau_{t}\right) \cdot \tau_{t}+\partial_{\sigma} R_{t} \nabla R_{t} \cdot\left(\nabla \tau_{t} \tau_{t}\right) X_{t, \tau} d \mathcal{H}^{1} \\
& =\int_{\partial F_{t}} \frac{1}{2}\left(\partial_{\sigma} R_{t}\right)^{2} \operatorname{div}_{\tau} X_{t}-\partial_{\sigma \sigma} R_{t} \dot{R}_{t}-\left(\partial_{\sigma \sigma} R_{t}\right)^{2} \partial_{\nu_{t}} R_{t}-k_{t}\left(\partial_{\sigma} R_{t}\right)^{2} \partial_{\sigma \sigma} R_{t} d \mathcal{H}^{1} \\
& +\int_{\partial F_{t}} \partial_{\sigma} R_{t}\left(\nabla^{2} R_{t} \tau_{t}\right) \cdot \tau_{t} X_{t, \tau}+\partial_{\sigma} R_{t} \nabla R_{t} \cdot\left(\nabla \tau_{t} \tau_{t}\right) X_{t, \tau} d \mathcal{H}^{1}
\end{aligned}
$$

Note that

$$
\frac{1}{2} \operatorname{div}_{\tau}\left(\left(\partial_{\sigma} R_{t}\right)^{2} X_{t}\right)=\frac{1}{2}\left(\partial_{\sigma} R_{t}\right)^{2} \operatorname{div}_{\tau} X_{t}+\partial_{\sigma} R_{t}\left(\nabla^{2} R_{t} \tau_{t}\right) \cdot \tau_{t} X_{t, \tau}+\partial_{\sigma} R_{t} \nabla R_{t} \cdot\left(\nabla \tau_{t} \tau_{t}\right) X_{t, \tau}
$$

Hence, using also (2.1), we get

$$
\begin{aligned}
\frac{d}{d t} & \left(\frac{1}{2} \int_{\partial F_{t}}\left(\partial_{\sigma} R_{t}\right)^{2} d \mathcal{H}^{1}\right) \\
& =\int_{\partial F_{t}} \frac{1}{2} \operatorname{div}_{\tau}\left(\left(\partial_{\sigma} R_{t}\right)^{2} X_{t}\right) d \mathcal{H}^{1}-\partial_{\sigma \sigma} R_{t} \dot{R}_{t}-\left(\partial_{\sigma \sigma} R_{t}\right)^{2} \partial_{\nu_{t}} R_{t}-k_{t}\left(\partial_{\sigma} R_{t}\right)^{2} \partial_{\sigma \sigma} R_{t} d \mathcal{H}^{1} \\
& =-\int_{\partial F_{t}} \partial_{\sigma \sigma} R_{t} \dot{R}_{t}+\left(\partial_{\sigma \sigma} R_{t}\right)^{2} \partial_{\nu_{t}} R_{t}+\frac{1}{2} k_{t}\left(\partial_{\sigma} R_{t}\right)^{2} \partial_{\sigma \sigma} R_{t} d \mathcal{H}^{1}
\end{aligned}
$$

Therefore, recalling (3.7), by (3.10) and (3.12) we get from (3.14) that 


$$
\begin{aligned}
\frac{d}{d t}\left(\frac{1}{2} \int_{\partial F_{t}}\left(\partial_{\sigma} R_{t}\right)^{2} d \mathcal{H}^{1}\right)=-\int_{\partial F_{t}} g\left(\nu_{t}\right)\left(\partial_{\sigma \sigma \sigma} R_{t}\right)^{2} d \mathcal{H}^{1}-\int_{\partial F_{t}} \partial_{\sigma \sigma} R_{t}\left(\dot{f}_{t} \circ \pi_{G}\right) d \mathcal{H}^{1} \\
-\int_{\partial F_{t}}\left(\partial_{\nu_{t}}\left(f_{t} \circ \pi_{G}\right)\left(\partial_{\sigma \sigma} R_{t}\right)^{2}-g\left(\nu_{t}\right) k_{t}^{2}\left(\partial_{\sigma \sigma} R_{t}\right)^{2}+\frac{1}{2} k_{t}\left(\partial_{\sigma} R_{t}\right)^{2} \partial_{\sigma \sigma} R_{t}\right) d \mathcal{H}^{1}
\end{aligned}
$$

By the ellipticity assumptions (2.8) we have that $c_{0} \leq g\left(\nu_{t}\right) \leq C_{0}$ and $\left|k_{t}\right| \leq \frac{1}{c_{0}}\left|k_{\varphi, t}\right| \leq C$, where $C$ depends also on the $C^{2, \alpha}$-norm of $h_{t}$. For $\varepsilon>0$ to be chosen, using also Young's inequality, we may estimate (3.15) as

$$
\begin{aligned}
& \frac{d}{d t}\left(\frac{1}{2} \int_{\partial F_{t}}\left(\partial_{\sigma} R_{t}\right)^{2} d \mathcal{H}^{1}\right)+c_{0} \int_{\partial F_{t}}\left(\partial_{\sigma \sigma \sigma} R_{t}\right)^{2} d \mathcal{H}^{1} \\
& \quad \leq C_{\varepsilon}\left\|\dot{f}_{t}\right\|_{H^{-\frac{1}{2}(\partial G)}}^{2}+\varepsilon\left\|\partial_{\sigma \sigma} R_{t}\right\|_{H^{\frac{1}{2}\left(\partial F_{t}\right)}}^{2}+C \int_{\partial F_{t}}\left(1+\left(\partial_{\sigma \sigma} R_{t}\right)^{2}+\left(\partial_{\sigma} R_{t}\right)^{2}\left|\partial_{\sigma \sigma} R_{t}\right|\right) d \mathcal{H}^{1},
\end{aligned}
$$

where the constant $C$ depends on the $C^{2, \alpha}$-norm of $h_{t}$ and the $C^{1, \alpha}$-norm of $f_{t}$. Since $\left\|\partial_{\sigma \sigma} R_{t}\right\|_{H^{\frac{1}{2}\left(\partial F_{t}\right)}} \leq C\left\|\partial_{\sigma \sigma \sigma} R_{t}\right\|_{L^{2}\left(\partial F_{t}\right)}$, by choosing $\varepsilon$ small enough we get

$$
\begin{aligned}
& \frac{d}{d t}\left(\frac{1}{2} \int_{\partial F_{t}}\left(\partial_{\sigma} R_{t}\right)^{2} d \mathcal{H}^{1}\right)+\frac{2}{3} c_{0} \int_{\partial F_{t}}\left(\partial_{\sigma \sigma \sigma} R_{t}\right)^{2} d \mathcal{H}^{1} \\
& \quad \leq C\left\|\dot{f}_{t}\right\|_{H^{-\frac{1}{2}(\partial G)}}^{2}+C \int_{\partial F_{t}}\left(1+\left(\partial_{\sigma \sigma} R_{t}\right)^{2}+\left(\partial_{\sigma} R_{t}\right)^{4}\right) d \mathcal{H}^{1} .
\end{aligned}
$$

Note now that by Theorem 6.1,

and

$$
\left\|\partial_{\sigma \sigma} R_{t}\right\|_{L^{2}}^{2} \leq C\left\|\partial_{\sigma \sigma \sigma} R_{t}\right\|_{L^{2}}\left\|\partial_{\sigma} R_{t}\right\|_{L^{2}} \leq \varepsilon\left\|\partial_{\sigma \sigma \sigma} R_{t}\right\|_{L^{2}}^{2}+\frac{C}{\varepsilon}\left\|\partial_{\sigma} R_{t}\right\|_{L^{2}}^{2}
$$

$$
\left\|\partial_{\sigma} R_{t}\right\|_{L^{4}}^{4} \leq C\left\|\partial_{\sigma \sigma \sigma} R_{t}\right\|_{L^{2}}^{\frac{1}{2}}\left\|\partial_{\sigma} R_{t}\right\|_{L^{2}}^{\frac{7}{2}} \leq \varepsilon\left\|\partial_{\sigma \sigma \sigma} R_{t}\right\|_{L^{2}}^{2}+\frac{C}{\varepsilon}\left\|\partial_{\sigma} R_{t}\right\|_{L^{2}}^{\frac{14}{3}}
$$

Hence the estimate (3.9) follows for $q=\frac{7}{3}$ by choosing $\varepsilon$ small enough.

Next theorem establishes the local in time existence for (3.6). The same result for $f=0$ is proved in [16]. The case considered here follows essentially from the same argument.

Theorem 3.4. Let $h_{0} \in H^{4}(\partial G)$ and let $f \in C^{\infty}(\partial G \times[0,+\infty))$. Then, there exist $\delta>0$ and $T>0$ such that if $\left\|h_{0}\right\|_{\left.C^{1}(\partial G)\right)} \leq \delta$, then (3.6) admits a smooth solution $\left(F_{t}\right)_{t}$ defined for all $t \in(0, T)$. Moreover, setting $\partial F_{t}=\left\{x+h_{t}(x) \nu_{G}(x)\right\}$, we have that $\left(h_{t}\right)_{t} \in H^{1}\left(0, T ; L^{2}(\partial G)\right) \cap$ $L^{2}\left(0, T ; H^{4}(\partial G)\right)$. Finally, there exists $\bar{\delta} \in(0, \bar{\eta})$, where $\bar{\eta}$ is as in (2.2), depending only on $G$ and $\Omega$, such that if $\sup _{(0, T)}\left\|h_{t}-h_{0}\right\|_{C^{1}(\partial G)}<\bar{\delta}$, then the solution can be extended beyond the time $T$.

Proof. The proof goes exactly as the one of Theorem 2.5 of [16, taking into account the presence of the forcing term $f$. Note that as in [16] in the first part of the proof we can only conclude that the time $T$ depends on $\left\|h_{0}\right\|_{H^{4}}$ and on $\|f\|_{L^{2}\left(0, T ; H^{2}\right)}$. However, one can then argue as in the second part of proof of Theorem 2.5 of [16] to conclude that the $\bar{\delta}$ for which the extension property holds is independent of $\left\|h_{0}\right\|_{H^{4}}$ and $\|f\|_{L^{2}\left(0, T ; H^{2}\right)}$, as long as $f \in L^{2}\left(0, T ; H^{2}\right)$ (a property which is implied by our assumption on $f$ ). Finally, the $C^{\infty_{-}}$ regularity of the solution for $t>0$ follows by standard arguments (or arguing as in the proof of Theorem 3.8 below where in fact the more complicated equation (3.1) is dealt with). 
In the next lemma we use the monotone quantity (3.9) to deduce regularity estimates for the flow (3.6).

Lemma 3.5. Let $F_{0}$ be a smooth initial set and $h_{0} \in C^{\infty}(\partial G)$ the function representing $\partial F_{0}$ as in (3.5). Fix $M_{0}>0, \alpha \in\left(0, \frac{1}{2}\right), \delta_{1} \in(0, \bar{\delta})$, with $\bar{\delta}$ as in Theorem 3.4. There exist $\delta_{0}>0$ and $T_{0}$, depending on $M_{0}, \alpha$, and $\delta_{1}$, such that if $f \in C^{\infty}(\partial G \times[0,+\infty))$ satisfies

$$
\sup _{\left(0, T_{0}\right)}\left\|f_{t}\right\|_{C^{1, \alpha}(\partial G)} \leq M_{0} \quad \text { and } \quad \int_{0}^{T_{0}}\left\|\dot{f}_{t}\right\|_{H^{-\frac{1}{2}(\partial G)}}^{2} \leq M_{0}
$$

and if $\left\|h_{0}\right\|_{H^{3}(\partial G)} \leq M_{0},\left\|h_{0}\right\|_{L^{2}(\partial G)}<\delta_{0}$, then the flow (3.6) exists on $\left(0, T_{0}\right)$ and

$$
\sup _{\left(0, T_{0}\right)}\left\|h_{t}\right\|_{C^{2, \alpha}(\partial G)} \leq \delta_{1} \quad \text { and } \quad \sup _{\left(0, T_{0}\right)}\left\|\partial_{\sigma} R_{t}\right\|_{L^{2}\left(\partial F_{t}\right)}^{2} \leq 2 C_{1} M_{0}+\left\|\partial_{\sigma} R_{0}\right\|_{L^{2}\left(\partial F_{0}\right)}^{2},
$$

where $C_{1}$ is the constant appearing in Lemma 3.3.

Proof. We fix $\delta_{1}<\bar{\delta}$ and observe if $\delta_{0}>0$ is sufficiently small, $\left\|h_{0}\right\|_{H^{3}(\partial G)} \leq M_{0}$ and $\left\|h_{0}\right\|_{L^{2}(\partial G)}<\delta_{0}$, then from (6.3) we get $\left\|h_{0}\right\|_{C^{2, \alpha}(\partial G)}<\bar{\delta}-\delta_{1}$. In particular, by Theorem 3.4 the flow exists for a short time and as long as $\left\|h_{t}\right\|_{C^{2, \alpha}(\partial G)}<\delta_{1}$, since this implies that $\left\|h_{t}-h_{0}\right\|_{C^{1}(\partial G)}<\bar{\delta}$.

Let us denote by $T_{0}$ the maximal time such that

$$
\left\|h_{t}\right\|_{C^{2, \alpha}(\partial G)}<\delta_{1} \text { and }\left\|\partial_{\sigma} R_{t}\right\|_{L^{2}\left(\partial F_{t}\right)}^{2}<2 C_{1} M_{0}+\left\|\partial_{\sigma} R_{0}\right\|_{L^{2}\left(\partial F_{0}\right)}^{2} \quad \text { for all } t \in\left(0, T_{0}\right) .
$$

We want to show that if (3.16) is satisfied, then $T_{0}$ is bounded away from 0 by a constant depending only $M_{0}, \alpha$, and $\delta_{1}$. Thus, without loss of generality we may assume that $T_{0} \leq 1$, otherwise there is nothing to prove.

Assume first that $\left\|h_{T_{0}}\right\|_{C^{2, \alpha}(\partial G)}=\delta_{1}$. From (3.18), from the first inequality in (3.16) and from the assumption $\left\|h_{0}\right\|_{H^{3}(\partial G)} \leq M_{0}$ we conclude that

$$
\left\|\partial_{\sigma} R_{t}\right\|_{L^{2}\left(\partial F_{t}\right)} \leq C\left(M_{0}\right) \quad \text { for all } t \in\left(0, T_{0}\right) .
$$

In turn, using again the first inequality in (3.16) we get

$$
\left\|\partial_{\sigma} k_{\varphi, t}\right\|_{L^{2}\left(\partial F_{t}\right)}^{2} \leq C\left(M_{0}\right) \quad \text { for all } t \in\left(0, T_{0}\right) .
$$

Now, by the first inequality in (3.18), recalling also formula (2.7), we deduce that

$$
\left\|h_{t}\right\|_{H^{3}(\partial G)} \leq C\left(M_{0}\right) \quad \text { for all } t \in\left(0, T_{0}\right) .
$$

Moreover, we conclude by integrating (3.8) over $(0, t)$ and by (3.19) that

$$
\left\|h_{t}\right\|_{L^{2}(\partial G)}^{2} \leq C\left(M_{0}\right) T_{0}+C\left\|h_{0}\right\|_{L^{2}(\partial G)}^{2} \leq C\left(M_{0}\right) T_{0}+C \delta_{0}^{2} \quad \text { for all } t \in\left(0, T_{0}\right) .
$$

In turn, by (6.3) and by (3.20) we get

$$
\delta_{1}=\left\|h_{T_{0}}\right\|_{C^{2, \alpha}(\partial G)} \leq C\left(\left\|h_{T_{0}}\right\|_{H^{3}(\partial G)}^{\theta}\left\|h_{T_{0}}\right\|_{L^{2}(\partial G)}^{1-\theta}+\left\|h_{T_{0}}\right\|_{L^{2}(\partial G)}\right) \leq C\left(M_{0}\right)\left(\sqrt{T_{0}}+\delta_{0}\right)^{1-\theta},
$$

where $\theta$ depends only on $\alpha$. It is clear from the above inequality that if $\delta_{0}$ is sufficiently small we get that $T_{0}$ is bounded away from 0 .

Assume now that $y\left(T_{0}\right)=2 C_{1} M_{0}+y(0)$, where we have set $y(t):=\left\|R_{\sigma}\right\|_{L^{2}\left(\partial F_{t}\right)}^{2}$. By integrating (3.9) over the time interval $\left(0, T_{0}\right)$ and using the second inequality in (3.16) we get

$$
y\left(T_{0}\right) \leq y(0)+C_{1} M_{0}+C_{1} \int_{0}^{T_{0}}(1+y)^{q} d t .
$$


Now, using the second inequality in (3.18) we conclude that

$$
2 C_{1} M_{0}+y(0)=y\left(T_{0}\right) \leq y(0)+C_{1} M_{0}+C_{1} T_{0}\left(1+2 C_{1} M_{0}+y(0)\right)^{q} .
$$

From this estimate we get again that $T_{0}$ is bounded away from 0 . This concludes the proof of the lemma.

We will need the following result for the elastic equilibrium, which states that if $F, \widetilde{F} \in$ $\mathfrak{h}_{M}^{2, \alpha}(G)$ are $C^{2, \alpha}$-close, then the corresponding elastic equilibria are $C^{2, \alpha}$-close to each other. More precisely, we have the following lemma.

Lemma 3.6. Let $0<\alpha<\beta \leq 1, M>0$ and $k \in \mathbb{N}$. Then there exists $C>0$ such that if $F, \widetilde{F} \in \mathfrak{h}_{M}^{k, \beta}(G)$ we have

$$
\left\|u_{F} \circ \pi_{F}^{-1}-u_{\widetilde{F}} \circ \pi_{\widetilde{F}}^{-1}\right\|_{C^{k, \alpha}(\partial G)} \leq C\left\|h_{F}-h_{\widetilde{F}}\right\|_{C^{k, \alpha}(\partial G)} .
$$

Here, we recall that $u_{F}$ and $u_{\widetilde{F}}$ denote the elastic equilibria corresponding to $F$ and $\tilde{F}$, respectively, as defined in (2.10).

Proof. The case $k=1$ can be proved as in [22, Lemma 6.10]. We now assume $k \geq 2$.

Denote by $\mathcal{U}$ the open set in $C^{k, \alpha}(\partial G)$ defined as

$$
\mathcal{U}:=\left\{h \in C^{k, \alpha}(\partial G):\|h\|_{L^{\infty}(\partial G)}<2 \bar{\eta} / 3,\|h\|_{C^{k, \alpha}(\partial G)}<M^{\prime}\right\},
$$

where $M^{\prime}>0$ is chosen so large that $\mathfrak{h}_{M}^{k, \beta}(G) \subset \mathcal{U}$.

Given $h \in \mathcal{U}$, we denote by $u_{h}$ the solution to (2.10), with $F$ replaced by the bounded set $F_{h}$ whose boundary is given by the (normal) graph of $h$ over $\partial G$.

Fix $\psi \in C_{c}^{\infty}(\Omega), 0 \leq \psi \leq 1, \psi \equiv 1$ in $\left\{d_{G} \leq 2 \bar{\eta} / 3\right\}$, and $\operatorname{supp} \psi \subset \subset\left\{d_{G}<\bar{\eta}\right\}$ and notice that if $\|h\|_{C^{k, \alpha}(\partial G)} \leq \delta^{\prime}$, for $\delta^{\prime}$ sufficiently small, then the map $\Phi_{h}: \Omega \backslash F_{h} \rightarrow \Omega \backslash G$ of the form

$$
\Phi_{h}(x)=x-h\left(\pi_{G}(x)\right) \psi(x) \nu_{G}\left(\pi_{G}(x)\right)
$$

is a $C^{k, \alpha}$-diffeomorphism.

Then setting $v_{h}:=u_{h} \circ\left(\Phi_{h}\right)^{-1}$ one can see that $v_{h}$ is the solution to

$$
\begin{cases}\operatorname{div}\left(\mathbb{A}\left(y, h\left(\pi_{G}(y)\right), \partial_{\sigma} h\left(\pi_{G}(y)\right)\right) \nabla v\right)=0 & \text { in } \Omega \backslash G \\ \mathbb{A}\left(y, h(y), \partial_{\sigma} h(y)\right) \nabla v\left[\nu_{G}\right]=0 & \text { on } \partial G \\ v=w_{0} & \text { on } \partial_{D} \Omega,\end{cases}
$$

where the entries of the tensor valued function $\mathbb{A}$ are 4-th order polynomials in $h \circ \pi$ and $\partial_{\sigma} h \circ \pi$ with $C^{k-1, \alpha}$-coefficients. It is easily checked that the map $\mathcal{F}: \mathcal{U} \times C^{k, \alpha}(\Omega \backslash G) \rightarrow$ $C^{k-2, \alpha}(\Omega \backslash G) \times C^{k-1, \alpha}(\partial G)$ given by

$$
\mathcal{F}(h, v):=\left(\operatorname{div}\left(\mathbb{A}\left(y, h\left(\pi_{G}(y)\right), \partial_{\sigma} h\left(\pi_{G}(y)\right)\right) \nabla v\right), \mathbb{A}\left(y, h(y), \partial_{\sigma} h(y)\right) \nabla v\left[\nu_{G}\right]\right)
$$

is of class $C^{1}$. We now check the invertibility (with continuity of the inverse) of $\partial_{v} \mathcal{F}\left(h, v_{h}\right)$, which is a linear operator from $C^{k, \alpha}(\Omega \backslash G)$ to $C^{k-2, \alpha}(\Omega \backslash G) \times C^{k-1, \alpha}(\partial G)$. This amounts to showing that for every $f \in C^{k-2, \alpha}(\Omega \backslash G)$ and $g \in C^{k-1, \alpha}(\partial G)$ the system

$$
\begin{cases}\operatorname{div}\left(\mathbb{A}\left(y, h\left(\pi_{G}(y)\right), \partial_{\sigma} h\left(\pi_{G}(y)\right)\right) \nabla w\right)=f & \text { in } \Omega \backslash G \\ \mathbb{A}\left(y, h(y), \partial_{\sigma} h(y)\right) \nabla w\left[\nu_{G}\right]=g & \text { on } \partial G, \\ w=0 & \text { on } \partial_{D} \Omega,\end{cases}
$$


admits a unique solution $w \in C^{k, \alpha}(\Omega \backslash G)$ such that $\|w\|_{C^{k, \alpha}(\Omega \backslash G)} \leq C\left(\|f\|_{C^{k-2, \alpha}(\Omega \backslash G)}+\right.$ $\left.\|g\|_{C^{k-1, \alpha}(\partial G)}\right)$, with $C$ depending only on $k, \alpha, G$ and $\Omega$. This follows from the classical Schauder estimates for linear elliptic systems (see for instance [3]). Thus, since $\mathcal{F}\left(h, v_{h}\right)=0$, we may apply the Implicit Function Theorem (see [4, Theorem 2.3]) to deduce that there exists $\delta>0$ such that the map $h \mapsto \mathcal{S}(h):=v_{h}$ is of class $C^{1}$ from a $\delta$-neighborhood of $h$ in the $C^{k, \alpha}(\partial G)$-norm to $C^{k, \alpha}(\Omega \backslash G)$.

Since $\mathcal{S}$ is $C^{1}$ in $\mathcal{U}$ and $\mathfrak{h}_{M}^{k, \beta}(G) \subset \mathcal{U}$ is compact in $C^{k \alpha}(\partial G)$, the Fréchet derivative $D \mathcal{S}(h)$ is equibounded in $\mathcal{L}\left(C^{k, \alpha}(\partial G) ; C^{k, \alpha}(\Omega \backslash G)\right)$ for $h \in \mathfrak{h}_{M}^{k, \beta}(G)$. Hence (3.21) easily follows.

Let us consider the flow (3.6), where $f$ satisfies the assumptions of Lemma 3.5. For every given time $t$ we consider the elastic equilibrium $u_{t}$ defined in (3.2). We start by observing that arguing as in [25, Theorem 3.2] one can show that $\dot{u}_{t}$ satisfies

$$
\int_{\Omega \backslash F_{t}} \mathbb{C} E\left(\dot{u}_{t}\right): E(\varphi) d x=-\int_{\partial F_{t}} \operatorname{div}_{\tau}\left(\partial_{\sigma \sigma} R_{t} \mathbb{C} E\left(u_{t}\right)\right) \cdot \varphi d \mathcal{H}^{1}
$$

for all $\varphi \in H^{1}\left(\Omega \backslash F_{t} ; \mathbb{R}^{2}\right)$ such that $\varphi=0$ on $\partial_{D} \Omega$. Note also that $\dot{u}_{t}=0$ on $\partial_{D} \Omega$.

We can now prove the regularity for the elastic equilibria $u_{t}$.

Lemma 3.7. Let $F_{0}$ and $\alpha$ be as in Lemma 3.5. Fix

$$
M_{0}>2\left\|Q\left(E\left(u_{G}\right)\right)\right\|_{C^{1, \alpha}(\partial G)} .
$$

There exist $\delta_{1} \in(0, \bar{\delta}), T_{0}>0$ and $\delta_{0}>0$ such that if $f$ satisfies (3.16), $\left\|h_{0}\right\|_{H^{3}(\partial G)} \leq M_{0}$ and $\left\|h_{0}\right\|_{L^{2}(\partial G)}<\delta_{0}$, then the flow (3.6) exists on $\left(0, T_{0}\right)$, (3.17) holds true and for every $t \in\left(0, T_{0}\right)$

$$
\left\|Q\left(E\left(u_{t}\right)\right) \circ \pi_{F_{t}}^{-1}\right\|_{C^{1, \alpha}(\partial G)}<M_{0} \quad \text { and } \quad \int_{0}^{T_{0}}\left\|\partial_{t}\left(Q\left(E\left(u_{t}\right)\right) \circ \pi_{F_{t}}^{-1}\right)\right\|_{H^{-\frac{1}{2}(\partial G)}}^{2} d t<M_{0} .
$$

Proof. Let $u_{G}$ be the elastic equilibrium in $G$. We first recall that if $\delta_{0}$ is as in Lemma 3.5, then by the first inequality in (3.17) and (3.21) we have

$$
\left\|u_{t} \circ \pi_{F_{t}}^{-1}-u_{G}\right\|_{C^{2, \alpha}(\partial G)} \leq C\left\|h_{t}\right\|_{C^{2, \alpha}(\partial G)} \leq C \delta_{1} .
$$

Therefore, choosing $\delta_{1} \in(0, \bar{\delta})$ sufficiently small (depending on $M_{0}$ ) and recalling (3.23), the first estimate in (3.24) follows.

For the second estimate we calculate

$$
\begin{aligned}
& \partial_{t}\left(Q\left(E\left(u_{t}\right)\right)\left(x+h_{t}(x) \nu_{G}(x)\right)\right) \\
& \quad=\mathbb{C} E\left(u_{t}\right) \circ \pi_{F_{t}}^{-1}:\left(\left(\nabla E\left(u_{t}\right) \circ \pi_{F_{t}}^{-1}\right)\left[\dot{h}_{t} \nu_{G}\right]\right)+\left(\mathbb{C} E\left(u_{t}\right): E\left(\dot{u}_{t}\right)\right) \circ \pi_{F_{t}}^{-1} .
\end{aligned}
$$

Therefore by the $C^{2, \alpha}$-bound (3.17) on $h_{t}$ and by (3.25) we have that

$$
\left\|\partial_{t}\left(Q\left(E\left(u_{t}\right)\right) \circ \pi_{F_{t}}^{-1}\right)\right\|_{H^{-\frac{1}{2}(\partial G)}} \leq C\left(M_{0}\right)\left\|\dot{h}_{t}\right\|_{L^{2}(\partial G)}+C\left(M_{0}\right)\left\|\mathbb{C} E\left(u_{t}\right): E\left(\dot{u}_{t}\right)\right\|_{H^{-\frac{1}{2}}\left(\partial F_{t}\right)}
$$

Observe first that the normal velocity of $\partial F_{t}$ can be written on $\partial G$ as

$$
V \circ \pi_{F_{t}}^{-1}=\dot{h}_{t} \frac{1+h_{t} k_{G}}{J_{t}} .
$$

Therefore by the definition of the flow, recalling the interpolation inequality (6.2), we get

$$
\left\|\dot{h}_{t}\right\|_{L^{2}(\partial G)} \leq C\|V\|_{L^{2}\left(\partial F_{t}\right)}=C\left\|\partial_{\sigma \sigma} R_{t}\right\|_{L^{2}\left(\partial F_{t}\right)} \leq C\left\|\partial_{\sigma \sigma \sigma} R_{t}\right\|_{L^{2}\left(\partial F_{t}\right)}^{\frac{1}{2}}\left\|\partial_{\sigma} R_{t}\right\|_{L^{2}\left(\partial F_{t}\right)}^{\frac{1}{2}} .
$$


In order to estimate second term in (3.26) we recall that $\mathbb{C} E\left(u_{t}\right)[\nu]=0$. Therefore, using (3.25) again, we get

$$
\begin{aligned}
\left\|\mathbb{C} E\left(u_{t}\right): E\left(\dot{u}_{t}\right)\right\|_{H^{-\frac{1}{2}\left(\partial F_{t}\right)}} & =\left\|\mathbb{C} E\left(u_{t}\right): \nabla \dot{u}_{t}\right\|_{H^{-\frac{1}{2}}\left(\partial F_{t}\right)} \\
& =\left\|\mathbb{C} E\left(u_{t}\right): \nabla_{\tau} \dot{u}_{t}\right\|_{H^{-\frac{1}{2}\left(\partial F_{t}\right)}} \leq C\left\|\nabla_{\tau} \dot{u}_{t}\right\|_{H^{-\frac{1}{2}}\left(\partial F_{t}\right)} .
\end{aligned}
$$

Choosing as a test function $\varphi=\dot{u}_{t}$ in the equation (3.22) we get arguing as above that

$$
\begin{aligned}
2 \int_{F_{t}} Q\left(E\left(\dot{u}_{t}\right)\right) d x & =-\int_{\partial F_{t}} \operatorname{div}_{\tau}\left(\partial_{\sigma \sigma} R_{t} \mathbb{C} E\left(u_{t}\right)\right) \cdot \dot{u}_{t} d \mathcal{H}^{1} \\
& =\int_{\partial F_{t}} \partial_{\sigma \sigma} R_{t} \mathbb{C} E\left(u_{t}\right): \nabla_{\tau} \dot{u}_{t} d \mathcal{H}^{1} \\
& \leq C\left(M_{0}\right)\left\|\partial_{\sigma \sigma} R_{t}\right\|_{H^{\frac{1}{2}}\left(\partial F_{t}\right)}\left\|\nabla_{\tau} \dot{u}_{t}\right\|_{H^{-\frac{1}{2}}\left(\partial F_{t}\right)} .
\end{aligned}
$$

Recall that $\dot{u}_{t}=0$ on $\partial_{D} \Omega$. Therefore by Korn's inequality, by (3.29) and by the interpolation inequality (6.1) we get

$$
\begin{aligned}
\left\|\nabla_{\tau} \dot{u}_{t}\right\|_{H^{-\frac{1}{2}\left(\partial F_{t}\right)}}^{2} & \leq C\left\|\dot{u}_{t}\right\|_{H^{\frac{1}{2}\left(\partial F_{t}\right)}}^{2} \leq C \int_{\Omega \backslash F_{t}}\left|\nabla \dot{u}_{t}\right|^{2} d x \\
& \leq C \int_{F_{t}} Q\left(E\left(\dot{u}_{t}\right)\right) d x \leq C\left(M_{0}\right)\left\|\partial_{\sigma \sigma} R_{t}\right\|_{H^{\frac{1}{2}\left(\partial F_{t}\right)}}\left\|\nabla_{\tau} \dot{u}_{t}\right\|_{H^{-\frac{1}{2}\left(\partial F_{t}\right)}} \\
& \leq C\left(M_{0}\right)\left(\left\|\partial_{\sigma \sigma \sigma} R_{t}\right\|_{L^{2}}^{\frac{3}{4}}\left\|\partial_{\sigma} R_{t}\right\|_{L^{2}}^{\frac{1}{4}}+\left\|\partial_{\sigma} R_{t}\right\|_{L^{2}}\right)\left\|\nabla_{\tau} \dot{u}_{t}\right\|_{H^{-\frac{1}{2}\left(\partial F_{t}\right)}} .
\end{aligned}
$$

Note that the first inequality above uses the fact that the tangential derivative is a continuous operator from $H^{1 / 2}$ to $H^{-1 / 2}$. This is a well-known fact, see for instance [25, Theorem 8.6]. This inequality together with (3.28) yields

$$
\left\|\mathbb{C} E\left(u_{t}\right): E\left(\dot{u}_{t}\right)\right\|_{H^{-\frac{1}{2}\left(\partial F_{t}\right)}} \leq C\left(M_{0}\right)\left(\left\|\partial_{\sigma \sigma \sigma} R_{t}\right\|_{L^{2}\left(\partial F_{t}\right)}^{\frac{3}{4}}\left\|\partial_{\sigma} R_{t}\right\|_{L^{2}\left(\partial F_{t}\right)}^{\frac{1}{4}}+\left\|\partial_{\sigma} R_{t}\right\|_{L^{2}\left(\partial F_{t}\right)}\right) .
$$

By combining the estimates (3.26), (3.27) and (3.30) we deduce that for every $\varepsilon>0$

$$
\int_{0}^{T_{0}}\left\|\left(\partial_{t} Q\left(E\left(u_{t}\right)\right)\right) \circ \pi_{F_{t}}^{-1}\right\|_{H^{-\frac{1}{2}(\partial G)}}^{2} d t \leq \int_{0}^{T_{0}}\left(\varepsilon\left\|\partial_{\sigma \sigma \sigma} R_{t}\right\|_{L^{2}\left(\partial F_{t}\right)}^{2}+C_{\varepsilon}\left\|\partial_{\sigma} R_{t}\right\|_{L^{2}\left(\partial F_{t}\right)}^{2}\right) d t .
$$

Hence, taking $\varepsilon$ sufficiently small we obtain by (3.9) and by Lemma 3.5

$$
\begin{aligned}
\int_{0}^{T_{0}}\left\|\left(\partial_{t} Q\left(E\left(u_{t}\right)\right)\right) \circ \pi_{F_{t}}^{-1}\right\|_{H^{-\frac{1}{2}(\partial G)}}^{2} d t \leq \\
\quad \leq \frac{1}{2} \int_{0}^{T_{0}}\left\|\dot{f}_{t}\right\|_{H^{-\frac{1}{2}(\partial G)}}^{2} d t+C\left(M_{0}\right) \sup _{\left(0, T_{0}\right)}\left(1+\left\|\partial_{\sigma} R_{t}\right\|_{L^{2}\left(\partial F_{t}\right.}^{2}\right)^{q} T_{0} \leq \frac{1}{2} M_{0}+C T_{0} .
\end{aligned}
$$

Thus the second estimate in (3.24) follows by choosing $T_{0}$ sufficiently small.

Proof of Theorem 3.2. We divide the proof into several steps.

Step 1. Fix $\mu \in(0,1)$ and let $M_{0}, T_{0}, \alpha, \delta_{1}$ and $\delta_{0}$ be as in Lemma 3.7. Let $f_{1}, f_{2} \in C^{\infty}(\partial G \times$ $[0,+\infty)$ satisfy the assumptions of Lemma 3.7, let $h_{0} \in C^{\infty}(\partial G)$ satisfy $\left\|h_{0}\right\|_{H^{3}(\partial G)} \leq M_{0}$, $\left\|h_{0}\right\|_{L^{2}(\partial G)}<\delta_{0}$, and let $F_{t, i}$ be a solution of (3.6) with $f$ replaced by $f_{i}$. Denote by $h_{t, i}$ the 
function such that $\partial F_{t, i}=\left\{x+h_{t, i}(x) \nu_{G}(x): x \in \partial G\right\}$. We start by showing that there exists $T \in\left(0, T_{0}\right)$ such that

$$
\int_{0}^{T} \int_{\partial G}\left(h_{t, 2}-h_{t, 1}\right)^{2} d \mathcal{H}^{1} d t \leq \mu \int_{0}^{T} \int_{\partial G}\left(f_{2}-f_{1}\right)^{2} d \mathcal{H}^{1} d t .
$$

Note in particular that the above inequality implies the uniqueness of the solution of (3.6) when all the data are smooth.

Recall that by Lemma 3.5 we have that $\left\|h_{t, i}\right\|_{C^{2, \alpha}} \leq \delta_{1}$ for all $t \in\left(0, T_{0}\right)$, for $i=1,2$. Note that we may write the equation (3.6) as

$$
\frac{\left(1+h_{t, i} k_{G}\right)}{J_{t, i}} \dot{h}_{t, i}=\frac{1}{J_{t, i}} \partial_{\sigma}\left(\frac{1}{J_{t, i}} \partial_{\sigma}\left(\left(g\left(\nu_{F_{t, i}}\right) k_{F_{t, i}}\right) \circ \pi_{F_{t, i}}^{-1}+f_{i}\right)\right) \quad \text { on } \partial G
$$

for $i=1,2$ respectively, where $J_{t, i}=\sqrt{\left(1+h_{t, i} k_{G}\right)^{2}+\left(\partial_{\sigma} h_{t, i}\right)^{2}}$. To simplify the notation we write $g_{t, i}$ and $k_{t, i}$ in place of $g\left(\nu_{F_{t, i}}\right)$ and $k_{F_{t, i}}$, respectively. Note that by the $C^{2, \alpha}$ bounds on $h_{t, 2}$ and $h_{t, 1}$, and by the expressions (2.5) and (2.6) we may estimate

$$
\left|g_{t, 2}\left(\pi_{F_{t, 2}}^{-1}(x)\right)-g_{t, 1}\left(\pi_{F_{t, 1}}^{-1}(x)\right)\right| \leq C\left(\left|\partial_{\sigma}\left(h_{t, 2}-h_{t, 1}\right)(x)\right|+\left|\left(h_{t, 2}-h_{t, 1}\right)(x)\right|\right)
$$

for every $x \in \partial G$. Moreover from the expression (2.7), from the $C^{2, \alpha}$-bounds on $h_{2}$ and $h_{1}$, and from the ellipticity assumptions on $\varphi$ we deduce that there are positive constants $c$ and $C$ such that

$$
\begin{aligned}
& {\left[k_{t, 2}\left(\pi_{F_{t, 2}}^{-1}\right)-k_{t, 1}\left(\pi_{F_{t, 1}}^{-1}\right)\right] } \partial_{\sigma \sigma}\left(h_{t, 2}-h_{t, 1}\right) \\
& \leq-c\left|\partial_{\sigma \sigma}\left(h_{t, 2}-h_{t, 1}\right)\right|^{2}+C\left(\left|\partial_{\sigma}\left(h_{t, 2}-h_{t, 1}\right)\right|^{2}+\left|\left(h_{t, 2}-h_{t, 1}\right)\right|^{2}\right)
\end{aligned}
$$

on $\partial G$.

Multiply the equation (3.32) by $h_{t, 2}-h_{t, 1}$ for $i=1,2$, integrate over $\partial G$ and integrate by parts twice to get

$$
\begin{aligned}
\int_{\partial G} \dot{h}_{t, i} & \left(h_{t, 2}-h_{t, 1}\right) d \mathcal{H}^{1} \\
= & \int_{\partial G} \partial_{\sigma}\left(\frac{1}{J_{t, i}} \partial_{\sigma}\left(\left(g_{t, i} k_{t, i}\right) \circ \pi_{F_{t, i}}^{-1}+f_{i}\right)\right)\left(\frac{1}{1+h_{t, i} k_{G}}\left(h_{t, 2}-h_{t, 1}\right)\right) d \mathcal{H}^{1} \\
& =\int_{\partial G}\left(\left(g_{t, i} k_{t, i}\right) \circ \pi_{F_{t, i}}^{-1}+f_{i}\right) \partial_{\sigma}\left(\frac{1}{J_{t, i}} \partial_{\sigma}\left(\frac{1}{1+h_{t, i} k_{G}}\left(h_{t, 2}-h_{t, 1}\right)\right)\right) d \mathcal{H}^{1} .
\end{aligned}
$$

Substract the equation for $i=1$ from the equation for $i=2$ to get

$$
\begin{aligned}
\frac{d}{d t}\left(\frac{1}{2} \int_{\partial G}(\right. & \left.\left.h_{t, 2}-h_{t, 1}\right)^{2} d \mathcal{H}^{1}\right)=\int_{\partial G}\left(h_{t, 2}-h_{t, 1}\right)\left(\dot{h}_{t, 2}-\dot{h}_{t, 1}\right) d \mathcal{H}^{1} \\
= & \int_{\partial G}\left(\left(g_{t, 2} k_{t, 2}\right) \circ \pi_{F_{t, 2}}^{-1}+f_{2}\right) \partial_{\sigma}\left(\frac{1}{J_{t, 2}} \partial_{\sigma}\left(\frac{1}{1+h_{t, 2} k_{G}}\left(h_{t, 2}-h_{t, 1}\right)\right)\right) d \mathcal{H}^{1} \\
& -\int_{\partial G}\left(\left(g_{t, 1} k_{t, 1}\right) \circ \pi_{F_{t, 1}}^{-1}+f_{1}\right) \partial_{\sigma}\left(\frac{1}{J_{t, 1}} \partial_{\sigma}\left(\frac{1}{1+h_{t, 1} k_{G}}\left(h_{t, 2}-h_{t, 1}\right)\right)\right) d \mathcal{H}^{1} .
\end{aligned}
$$


By the $C^{2, \alpha}$-bounds on $h_{t, 2}, h_{t, 1}$, by $C^{0, \alpha}$-bounds on $f_{2}, f_{1}$, by (2.7) and by (3.33) and (3.34) we conclude that there are positive constants $c$ and $C$ such that

$$
\begin{aligned}
\frac{d}{d t}\left(\frac { 1 } { 2 } \int _ { \partial G } \left(h_{t, 2}\right.\right. & \left.\left.-h_{t, 1}\right)^{2} d \mathcal{H}^{1}\right)+c \int_{\partial G}\left|\partial_{\sigma \sigma}\left(h_{t, 2}-h_{t, 1}\right)\right|^{2} d \mathcal{H}^{1} \\
& \leq C \int_{\partial G}\left(f_{2}-f_{1}\right)^{2} d \mathcal{H}^{1}+C \int_{\partial G}\left|\partial_{\sigma}\left(h_{t, 2}-h_{t, 1}\right)\right|^{2}+\left(h_{t, 2}-h_{t, 1}\right)^{2} d \mathcal{H}^{1}
\end{aligned}
$$

Denote $w_{t}(x):=h_{t, 2}(x)-h_{t, 1}(x)$. By interpolation we have

$$
\left\|\partial_{\sigma} w_{t}\right\|_{L^{2}}^{2} \leq C\left\|\partial_{\sigma \sigma} w_{t}\right\|_{L^{2}}\left\|w_{t}\right\|_{L^{2}}+C\left\|w_{t}\right\|_{L^{2}}^{2} \leq \varepsilon\left\|\partial_{\sigma \sigma} w_{t}\right\|_{L^{2}}^{2}+C_{\varepsilon}\left\|w_{t}\right\|_{L^{2}}^{2} .
$$

Hence, for $\varepsilon$ small enough, we obtain by (3.35) that

$$
\frac{d}{d t}\left(\int_{\partial G} w_{t}^{2} \mathcal{H}^{1}\right) \leq C \int_{\partial G} w_{t}^{2} d \mathcal{H}^{1}+C \int_{\partial G}\left(f_{2}-f_{1}\right)^{2} d \mathcal{H}^{1} .
$$

Take $T \in\left(0, T_{0}\right)$. Recall that $w_{0} \equiv 0$. Therefore integrating (3.36) over $(0, t)$, with $t \in(0, T)$, implies

$$
\int_{\partial G} w_{t}^{2} d \mathcal{H}^{1} \leq C \int_{0}^{T} \int_{\partial G} w_{s}^{2} d \mathcal{H}^{1} d s+C \int_{0}^{T} \int_{\partial G}\left(f_{2}-f_{1}\right)^{2} d \mathcal{H}^{1} d s .
$$

Integrating (3.37) over $(0, T)$ yields

$$
\int_{0}^{T} \int_{\partial G} w_{t}^{2} d \mathcal{H}^{1} d t \leq C T \int_{0}^{T} \int_{\partial G} w_{t}^{2} d \mathcal{H}^{1} d t+C T \int_{0}^{T} \int_{\partial G}\left(f_{2}-f_{1}\right)^{2} d \mathcal{H}^{1} d t .
$$

Therefore (3.31) follows by taking $T \in\left(0, T_{0}\right)$ sufficiently small.

Step 2. Fix $M_{0}>2\left\|Q\left(E\left(u_{G}\right)\right)\right\|_{C^{1, \alpha}(\partial G)}, \mu \in(0,1)$, and let $T, \delta_{0}$ be as in Step 1. Define the function space

$$
\mathcal{C}:=\left\{f \in L^{2}\left(0, T ; L^{2}(\partial G)\right): \sup _{(0, T)}\|f\|_{C^{1, \alpha}} \leq M_{0}, \int_{0}^{T}\left\|\dot{f}_{t}\right\|_{H^{-\frac{1}{2}(\partial G)}}^{2} \leq M_{0}\right\} .
$$

We want to show that for every $f \in \mathcal{C}$ equation (3.6) has a solution in the interval $(0, T)$, and that (3.31) holds for $f_{1}, f_{2} \in \mathcal{C}$.

To this end, fix $h_{0} \in H^{3}(\partial G)$ satisfying $\left\|h_{0}\right\|_{H^{3}(\partial G)} \leq M_{0},\left\|h_{0}\right\|_{L^{2}(\partial G)}<\delta_{0}$, and let $f \in \mathcal{C}$. Consider a sequence $f_{n} \in \mathcal{C} \cap C^{\infty}(\partial G \times[0,+\infty))$ such that $f_{n} \rightarrow f$ in $L^{2}\left(0, T ; L^{2}(\partial G)\right)$ and a sequence of smooth functions $h_{n}$ such that $\left\|h_{n}\right\|_{H^{3}(\partial G)} \leq M_{0}$ and $h_{n} \rightarrow h_{0}$ in $L^{2}(\partial G)$. Denote by $F_{t, n}$ the solution of (3.6) with forcing term $f_{n}$ and initial datum $h_{n}$, and let $h_{t, n}$ be the function on $\partial G$ such that $\partial F_{t, n}=\left\{x+h_{t, n}(x) \nu_{G}(x): x \in \partial G\right\}$.

Observe that from (3.17) we have that

$$
\sup _{n} \sup _{(0, T)}\left(\left\|h_{t, n}\right\|_{C^{2, \alpha}(\partial G)}+\left\|\partial_{\sigma} R_{t, n}\right\|_{L^{2}\left(\partial F_{t}\right)}\right)<+\infty,
$$

where $R_{t, n}$ is defined as in (3.7) with $f$ replaced by $f_{n}$. In turn (3.9) yields that $R_{t, n}$ is uniformly bounded in $L^{2}\left(0, T ; H^{3}(\partial G)\right)$ and thus $h_{t, n}$ is uniformly bounded in $H^{1}\left(0, T ; H^{1}(\partial G)\right)$. Therefore, up to a (not relabelled) subsequence, we may assume that $h_{t, n} \rightarrow h_{t}$ weakly in $H^{1}\left(0, T ; H^{1}(\partial G)\right)$ and, recalling the uniform $C^{2, \alpha}$ bounds on $h_{t, n}$ we may conclude that in fact $h_{t, n} \rightarrow h_{t}$ in $C^{2, \beta}(\partial G)$ for all $\beta \in(0, \alpha)$ and for all $t \in(0, T)$ and thus $R_{t, n} \circ \pi_{F_{t, n}}^{-1} \rightarrow R_{t} \circ \pi_{F_{t}}^{-1}$ in $C^{0, \beta}(\partial G)$, where $F_{t}$ is the set corresponding to $h_{t}$. It is now easy to see that the equation passes to the limit and $F_{t}$ is a solution of (3.6) with initial datum $h_{0}$ and forcing term $f$. 
Note also that the same approximation argument yields that (3.31) holds true also in the case where $f_{1}, f_{2} \in \mathcal{C}$, so that in particular the solution is unique also in this case. Moreover, again by approximation, the conclusions of Lemmas 3.5 and 3.7 remain true.

Step 3. Fix $h_{0}$ as in Step 2 and consider the map $\mathcal{T}: \mathcal{C} \rightarrow \mathcal{C}$ defined by $T f(\cdot, t)=-Q\left(E\left(u_{t}\right)\right) \circ$ $\pi_{F_{t}}^{-1}$ for all $t \in[0, T)$, where $F_{t}$ is the solution of (3.6) with initial datum $h_{0}$ and forcing term $f$ (and $u_{t}$ is the elastic equilibrium in $\Omega \backslash F_{t}$ ). From (3.24), which holds also in our case thanks to the previous step, it follows that the map is well defined. In order to conclude the proof it is enough to show that $\mathcal{T}$ is a contraction and thus it admits a fixed point. To this aim, with the same notation of Step 1, for any $f_{1}, f_{2} \in \mathcal{C}$ and for any $\varepsilon>0$ we have

$$
\begin{aligned}
& \int_{0}^{T}\left\|Q\left(E\left(u_{t, 1}\right)\right) \circ \pi_{F_{t, 1}}^{-1}-Q\left(E\left(u_{t, 2}\right)\right) \circ \pi_{F_{t, 2}}^{-1}\right\|_{L^{2}(\partial G)}^{2} d t \\
& \quad \leq C \int_{0}^{T}\left\|Q\left(E\left(u_{t, 1}\right)\right) \circ \pi_{F_{t, 1}}^{-1}-Q\left(E\left(u_{t, 2}\right)\right) \circ \pi_{F_{t, 2}}^{-1}\right\|_{C^{0, \alpha}(\partial G)}^{2} d t \\
& \quad \leq C \int_{0}^{T}\left\|h_{t, 1}-h_{t, 2}\right\|_{C^{1, \alpha}(\partial G)}^{2} d t \\
& \quad \leq C \int_{0}^{T}\left[\left\|\partial_{\sigma \sigma}\left(h_{t, 1}-h_{t, 2}\right)\right\|_{L^{2}}^{2 \theta}\left\|h_{t, 1}-h_{t, 2}\right\|_{L^{2}}^{2(1-\theta)}+\left\|h_{t, 1}-h_{t, 2}\right\|_{L^{2}}^{2}\right] d t \\
& \quad \leq \int_{0}^{T}\left[\varepsilon\left\|\partial_{\sigma \sigma}\left(h_{t, 1}-h_{t, 2}\right)\right\|_{L^{2}}^{2}+C_{\varepsilon}\left\|h_{t, 1}-h_{t, 2}\right\|_{L^{2}}^{2}\right] d t,
\end{aligned}
$$

where we used (3.21) and (6.3). We use (3.35) and (6.2), argue as in Step 1, to control the last integral in the above chain of inequalities and deduce that there exists $C_{1}>0$ independent of $\varepsilon$ such that

$$
\begin{aligned}
& \int_{0}^{T} \| Q\left(E\left(u_{t, 1}\right)\right) \circ \pi_{F_{t, 1}}^{-1}-Q\left(E\left(u_{t, 2}\right)\right) \circ \pi_{F_{t, 2}}^{-1} \|_{L^{2}(\partial G)}^{2} d t \\
& \leq C_{1} \varepsilon \int_{0}^{T}\left\|f_{1}-f_{2}\right\|_{L^{2}}^{2} d t+C_{\varepsilon} \int_{0}^{T}\left\|h_{t, 1}-h_{t, 2}\right\|_{L^{2}}^{2} d t \\
& \leq C_{1} \varepsilon \int_{0}^{T}\left\|f_{1}-f_{2}\right\|_{L^{2}}^{2} d t+C_{\varepsilon} \mu \int_{0}^{T}\left\|f_{1}-f_{2}\right\|_{L^{2}}^{2} d t
\end{aligned}
$$

where the last inequality follows from (3.31). The conclusion follows by taking $\varepsilon$ and then $\mu$ sufficiently small.

We conclude this section by showing that the solution provided by Theorem 3.2 is in fact classical, namely of class $C^{\infty}$.

Theorem 3.8. Under the assumptions of Theorem 3.2 we have $\left(h_{t}\right)_{t \in(0, T)} \in C^{\infty}\left(0, T ; C^{\infty}(\partial G)\right)$.

Proof. As in the proof of Theorem 3.2 we rewrite the equation on $\partial G$, see (3.32), thus getting

$$
\frac{\left(1+h_{t} k_{G}\right)}{J_{t}} \dot{h}_{t}=\frac{1}{J_{t}} \partial_{\sigma}\left(\frac{1}{J_{t}} \partial_{\sigma}\left(\left(g\left(\nu_{F_{t}}\right) k_{F_{t}}\right) \circ \pi_{F_{t}}^{-1}-Q_{t}\right)\right) \quad \text { on } \partial G,
$$

where we have set $Q_{t}:=Q\left(E\left(u_{t}\right)\right) \circ \pi_{F_{t}}^{-1}$. We divide the proof in four steps.

Step 1. Given $\Delta t \neq 0$, let us subtract the equation above at time $t$ from the same equation at time $t+\Delta t$ and multiply both sides by $h_{t+\Delta t}-h_{t}$. Then, integrating by part and arguing 
as in the proof of (3.35) we get, using also Proposition 6.1 to estimate $\left\|\partial_{\sigma}\left(h_{t+\Delta t}-h_{t}\right)\right\|_{L^{2}(\partial G)}$,

$$
\begin{aligned}
\frac{d}{d t}\left(\frac{1}{2} \int_{\partial G}\left(h_{t+\Delta t}-h_{t}\right)^{2} d \mathcal{H}^{1}\right) & +c \int_{\partial G}\left|\partial_{\sigma \sigma}\left(h_{t+\Delta t}-h_{t}\right)\right|^{2} d \mathcal{H}^{1} \\
& \leq C \int_{\partial G}\left(Q_{t+\Delta t}-Q_{t}\right)^{2} d \mathcal{H}^{1}+C \int_{\partial G}\left(h_{t+\Delta t}-h_{t}\right)^{2} d \mathcal{H}^{1}
\end{aligned}
$$

Fix now $\alpha \in\left(0, \frac{1}{2}\right)$. Using Proposition 6.1 and the estimate (3.21) with $F$ and $G$ replaced respectively by $F_{t+\Delta t}$ and $F_{t}$ we have

$$
\begin{aligned}
\left\|Q_{t+\Delta t}-Q_{t}\right\|_{L^{2}(\partial G)} & \leq C\left\|Q_{t+\Delta t}-Q_{t}\right\|_{C^{0, \alpha}(\partial G)} \leq C\left\|h_{t+\Delta t}-h_{t}\right\|_{C^{1, \alpha}(\partial G)} \\
& \leq C\left(\left\|\partial_{\sigma \sigma}\left(h_{t+\Delta t}-h_{t}\right)\right\|_{L^{2}(\partial G)}^{\vartheta}\left\|h_{t+\Delta t}-h_{t}\right\|_{L^{2}(\partial G)}^{1-\vartheta}+\left\|h_{t+\Delta t}-h_{t}\right\|_{L^{2}(\partial G)}\right) .
\end{aligned}
$$

Inserting this inequality in (3.39) we get

$$
\frac{d}{d t}\left(\frac{1}{2} \int_{\partial G}\left(h_{t+\Delta t}-h_{t}\right)^{2} d \mathcal{H}^{1}\right)+c \int_{\partial G}\left|\partial_{\sigma \sigma}\left(h_{t+\Delta t}-h_{t}\right)\right|^{2} d \mathcal{H}^{1} \leq C\left\|h_{t+\Delta t}-h_{t}\right\|_{L^{2}(\partial G)}^{2} .
$$

Then for $\mathcal{L}^{1}$-a.e. $t_{0}, t_{1}$ with $0<t_{0}<t_{1}<T$, integrating the above inequality in $\left(t_{0}, t_{1}\right)$, we have

$$
\begin{aligned}
\left\|h_{t_{1}+\Delta t}-h_{t_{1}}\right\|_{L^{2}(\partial G)}^{2}+\int_{t_{0}}^{t_{1}} & \left\|\partial_{\sigma \sigma}\left(h_{t+\Delta t}-h_{t}\right)\right\|_{L^{2}(\partial G)}^{2} d t \\
& \leq\left\|h_{t_{0}+\Delta t}-h_{t_{0}}\right\|_{L^{2}(\partial G)}^{2}+C \int_{t_{0}}^{t_{1}}\left\|h_{t+\Delta t}-h_{t}\right\|_{L^{2}(\partial G)}^{2} d t .
\end{aligned}
$$

Finally, dividing both sides of this inequality by $(\Delta t)^{2}$, letting $\Delta t \rightarrow 0$ and recalling that $h \in H^{1}\left(0, T ; H^{1}(\partial G)\right)$, we conclude that for any time interval $J \subset \subset(0, T)$

$$
\sup _{t \in J}\left\|\dot{h}_{t}\right\|_{L^{2}(\partial G)}^{2}+\int_{J}\left\|\partial_{t}\left(\partial_{\sigma \sigma} h_{t}\right)\right\|_{L^{2}(\partial G)}^{2} d t<\infty
$$

Step 2. We start again by subtracting equation (3.38) at time $t$ from the same equation at time $t+\Delta t$. We now multiply both sides by $\partial_{\sigma \sigma}\left(h_{t+\Delta t}-h_{t}\right)$. Then, arguing as in the proof of (3.39) we get the following estimate

$$
\begin{aligned}
\frac{d}{d t}\left(\frac{1}{2} \int_{\partial G}\left(\partial_{\sigma}\left(h_{t+\Delta t}-h_{t}\right)\right)^{2} d \mathcal{H}^{1}\right)+c \int_{\partial G}\left|\partial_{\sigma \sigma \sigma}\left(h_{t+\Delta t}-h_{t}\right)\right|^{2} d \mathcal{H}^{1} \\
\leq C \int_{\partial G}\left(\partial_{\sigma}\left(Q_{t+\Delta t}-Q_{t}\right)\right)^{2} d \mathcal{H}^{1}+C \int_{\partial G}\left(h_{t+\Delta t}-h_{t}\right)^{2} d \mathcal{H}^{1} .
\end{aligned}
$$

As in the previous step we may estimate, using (3.21) and Proposition 6.1,

$$
\begin{aligned}
\left\|\partial_{\sigma}\left(Q_{t+\Delta t}-Q_{t}\right)\right\|_{L^{2}(\partial G)} & \leq C\left\|\partial_{\sigma}\left(Q_{t+\Delta t}-Q_{t}\right)\right\|_{C^{0, \alpha}(\partial G)} \leq C\left\|h_{t+\Delta t}-h_{t}\right\|_{C^{2, \alpha}(\partial G)} \\
& \leq C\left\|\partial_{\sigma \sigma \sigma}\left(h_{t+\Delta t}-h_{t}\right)\right\|_{L^{2}}^{\vartheta}\left\|h_{t+\Delta t}-h_{t}\right\|_{L^{2}}^{1-\vartheta}+C\left\|h_{t+\Delta t}-h_{t}\right\|_{L^{2}} .
\end{aligned}
$$


Using this estimate and integrating (3.41) in $\left(t_{0}, t_{1}\right)$ for $\mathcal{L}^{1}$-a.e. $t_{0}, t_{1}$ with $0<t_{0}<t_{1}<T$, we have

$$
\begin{aligned}
\left\|\partial_{\sigma}\left(h_{t_{1}+\Delta t}-h_{t_{1}}\right)\right\|_{L^{2}(\partial G)}^{2} & +c \int_{t_{0}}^{t_{1}}\left\|\partial_{\sigma \sigma \sigma}\left(h_{t+\Delta t}-h_{t}\right)\right\|_{L^{2}(\partial G)}^{2} d t \\
& \leq\left\|\partial_{\sigma}\left(h_{t_{0}+\Delta t}-h_{t_{0}}\right)\right\|_{L^{2}(\partial G)}^{2}+C \int_{t_{0}}^{t_{1}}\left\|h_{t+\Delta t}-h_{t}\right\|_{L^{2}(\partial G)}^{2} d t
\end{aligned}
$$

Divide both sides of this inequality by $(\Delta t)^{2}$ and recall that $\partial_{\sigma \sigma \sigma} h_{t} \in L^{2}\left(0, T ; L^{2}(\partial G)\right)$ and that by (3.40) $\partial_{t}\left(\partial_{\sigma} h_{t}\right) \in L_{l o c}^{2}\left(0, T ; H^{1}(\partial G)\right)$. Using this information and letting $\Delta t \rightarrow 0$ we conclude that for every interval $J \subset \subset(0, T)$

$$
\sup _{t \in J}\left\|\partial_{t}\left(\partial_{\sigma} h_{t}\right)\right\|_{L^{2}(\partial G)}^{2}+\int_{J}\left\|\partial_{t}\left(\partial_{\sigma \sigma \sigma} h_{t}\right)\right\|_{L^{2}(\partial G)}^{2} d t<\infty .
$$

Note that from the previous inequality and by the equation (3.32) we have that for every interval $J \subset \subset(0, T)$

$$
\sup _{t \in J}\left(\left\|\partial_{\sigma \sigma \sigma} R_{t}\right\|_{L^{2}(\partial G)}+\left\|\partial_{\sigma \sigma \sigma} h_{t}\right\|_{L^{2}(\partial G)}\right)<\infty .
$$

In particular, from this inequality we deduce that

$$
\sup _{t \in J}\left(\left\|\partial_{\sigma} R_{t}\right\|_{C^{0, \alpha}(\partial G)}+\left\|h_{t}\right\|_{C^{2, \alpha}(\partial G)}\right)<\infty .
$$

In turn, since $\left\|\partial_{\sigma} Q_{t}\right\|_{C^{0, \alpha}(\partial G)} \leq C\left(G,\left\|h_{t}\right\|_{C^{2, \alpha}(\partial G)}\right)$, the above inequality implies immediately that

$$
\sup _{t \in J}\left\|h_{t}\right\|_{C^{3, \alpha}(\partial G)}<\infty
$$

Step 3. At this point we would like to continue as before, subtracting the equations (3.38) at times $t+\Delta t$ an $t$ and multiplying the resulting difference by $\partial_{\sigma \sigma \sigma \sigma}\left(h_{t+\Delta t}-h_{t}\right)$. However this argument only works provided we know that $h \in L_{l o c}^{2}\left(0, T ; H^{4}(\partial G)\right)$.

To prove this property of $h$ we go back to equation (3.38) and, denoting by $s$ the arclength on $\partial G$, we subtract the equation for $h$ from the same equation for $h(\cdot+\Delta s)$, where $\Delta s$ is a non zero increment of the arclength. Then we multiply both sides by $\partial_{\sigma \sigma} h(\cdot+\Delta s)-\partial_{\sigma \sigma} h$ to deduce with the usual calculations that

$$
\begin{aligned}
\frac{d}{d t}\left(\frac{1}{2} \int_{\partial G}\left(\partial_{\sigma}\left(h_{t}(\cdot+\Delta s)-h_{t}\right)\right)^{2} d \mathcal{H}^{1}\right)+c \int_{\partial G}\left|\partial_{\sigma \sigma \sigma}\left(h_{t}(\cdot+\Delta s)-h_{t}\right)\right|^{2} d \mathcal{H}^{1} \\
\quad \leq C \int_{\partial G}\left(\partial_{\sigma}\left(Q_{t}(\cdot+\Delta s)-Q_{t}\right)\right)^{2} d \mathcal{H}^{1}+C \int_{\partial G}\left(h_{t}(\cdot+\Delta s)-h_{t}\right)^{2} d \mathcal{H}^{1} .
\end{aligned}
$$

As before we estimate

$$
\begin{aligned}
\| \partial_{\sigma}\left(Q_{t}(\cdot+\Delta s)\right. & \left.\left.-Q_{t}\right)\left\|_{L^{2}(\partial G)} \leq C\right\| h_{t}(\cdot+\Delta s)-h_{t}\right) \|_{C^{2, \alpha}(\partial G)} \\
& \leq C\left\|\partial_{\sigma \sigma \sigma}\left(h_{t}(\cdot+\Delta s)-h_{t}\right)\right\|_{L^{2}}^{\vartheta}\left\|h_{t}(\cdot+\Delta s)-h_{t}\right\|_{L^{2}}^{1-\vartheta}+C\left\|h_{t}(\cdot+\Delta s)-h_{t}\right\|_{L^{2}}
\end{aligned}
$$

so to obtain that for $\mathcal{L}^{1}$-a.e. $t_{0}, t_{1}$ with $0<t_{0}<t_{1}<T$

$$
\begin{aligned}
\| \partial_{\sigma}\left(h_{t_{1}}(\cdot+\Delta s)-\right. & \left.h_{t_{1}}\right)\left\|_{L^{2}(\partial G)}^{2}+\int_{t_{0}}^{t_{1}}\right\| \partial_{\sigma \sigma \sigma}\left(h_{t}(\cdot+\Delta s)-h_{t}\right) \|_{L^{2}(\partial G)}^{2} d t \\
\leq & \left\|\partial_{\sigma}\left(h_{t_{0}}(\cdot+\Delta s)-h_{t_{0}}\right)\right\|_{L^{2}(\partial G)}^{2}+C \int_{t_{0}}^{t_{1}}\left\|h_{t}(\cdot+\Delta s)-h_{t}\right\|_{L^{2}(\partial G)}^{2} d t .
\end{aligned}
$$


Thus, we may conclude that for every interval $J \subset \subset(0, T)$

$$
\sup _{t \in J}\left\|\partial_{\sigma \sigma} h_{t}\right\|_{L^{2}(\partial G)}^{2}+\int_{J}\left\|\partial_{\sigma \sigma \sigma \sigma} h_{t}\right\|_{L^{2}(\partial G)}^{2} d t<\infty .
$$

We now use this estimate, together with the estimate (3.43) obtained in the previous step, in order to show (3.44) below.

To this end, we subtract equation (3.38) at time $t$ from the same equation at time $t+\Delta t$ and multiply both sides by $\partial_{\sigma \sigma \sigma \sigma}\left(h_{t+\Delta t}-h_{t}\right)$ and we obtain

$$
\begin{aligned}
\frac{d}{d t}\left(\frac{1}{2} \int_{\partial G}\left(\partial_{\sigma \sigma}\left(h_{t+\Delta t}-h_{t}\right)\right)^{2} d \mathcal{H}^{1}\right)+c \int_{\partial G}\left|\partial_{\sigma \sigma \sigma \sigma}\left(h_{t+\Delta t}-h_{t}\right)\right|^{2} d \mathcal{H}^{1} \\
\leq C \int_{\partial G}\left(\partial_{\sigma \sigma}\left(Q_{t+\Delta t}-Q_{t}\right)\right)^{2} d \mathcal{H}^{1}+C \int_{\partial G}\left(h_{t+\Delta t}-h_{t}\right)^{2} d \mathcal{H}^{1} .
\end{aligned}
$$

Then using, (3.43), (3.21) and Proposition 6.1, we have

$$
\begin{aligned}
\left\|\partial_{\sigma \sigma}\left(Q_{t+\Delta t}-Q_{t}\right)\right\|_{L^{2}(\partial G)} & \leq C\left\|\partial_{\sigma \sigma}\left(Q_{t+\Delta t}-Q_{t}\right)\right\|_{C^{0, \alpha}(\partial G)} \leq C\left\|h_{t+\Delta t}-h_{t}\right\|_{C^{3, \alpha}(\partial G)} \\
& \leq C\left\|\partial_{\sigma \sigma \sigma}\left(h_{t+\Delta t}-h_{t}\right)\right\|_{L^{2}}^{\vartheta}\left\|h_{t+\Delta t}-h_{t}\right\|_{L^{2}}^{1-\vartheta}+C\left\|h_{t+\Delta t}-h_{t}\right\|_{L^{2}} .
\end{aligned}
$$

Then, arguing as in the proof of (3.42), we get

$$
\sup _{t \in J}\left\|\partial_{t}\left(\partial_{\sigma \sigma} h_{t}\right)\right\|_{L^{2}(\partial G)}^{2}+\int_{J}\left\|\partial_{t}\left(\partial_{\sigma \sigma \sigma \sigma} h_{t}\right)\right\|_{L^{2}(\partial G)}^{2} d t<\infty .
$$

Then, arguing as in the proof of (3.43) we have that

$$
\sup _{t \in J}\left\|h_{t}\right\|_{C^{4, \alpha}(\partial G)}<\infty
$$

At this point we proceed by induction, obtaining at each step first an increment in the space regularity and then the corresponding estimate with respect to time. More precisely, for every interval $J \subset \subset(0, T)$ and every integer $k \geq 2$ we first have that

$$
\sup _{t \in J}\left\|\partial_{\sigma}^{k} h_{t}\right\|_{L^{2}(\partial G)}^{2}+\int_{J}\left\|\partial_{\sigma}^{k+2} h_{t}\right\|_{L^{2}(\partial G)}^{2} d t<\infty
$$

Then from this we deduce that again for every interval $J \subset \subset(0, T)$

$$
\sup _{t \in J}\left\|\partial_{t}\left(\partial_{\sigma}^{k} h_{t}\right)\right\|_{L^{2}(\partial G)}^{2}+\int_{J}\left\|\partial_{t}\left(\partial_{\sigma}^{k+2} h_{t}\right)\right\|_{L^{2}(\partial G)}^{2} d t<\infty
$$

and in turn that

$$
\sup _{t \in J}\left\|h_{t}\right\|_{C^{k+2, \alpha}(\partial G)}<\infty
$$

In conclusion this proves that $h \in W_{l o c}^{1, \infty}\left(0, T ; C^{\infty}(\partial G)\right)$.

Step 4. Let us now show the full regularity of $h$ with respect to time. As in Step 1 we fix $\Delta t \neq 0$ and subtract equation (3.38) from the same equation at time $t+\Delta t$. However, differently from before, we multiply both sides of this difference by $\dot{h}_{t+\Delta t}-\dot{h}_{t}$. Then, a simple use of Young's inequality and Proposition 6.1 yields

$$
\begin{aligned}
\int_{\partial G}\left(\dot{h}_{t+\Delta t}-\dot{h}_{t}\right)^{2} d \mathcal{H}^{1} & \leq C \int_{\partial G}\left|\partial_{\sigma \sigma \sigma \sigma}\left(h_{t+\Delta t}-h_{t}\right)\right|^{2} d \mathcal{H}^{1} \\
& +C \int_{\partial G}\left(\partial_{\sigma \sigma}\left(Q_{t+\Delta t}-Q_{t}\right)\right)^{2} d \mathcal{H}^{1}+C \int_{\partial G}\left(h_{t+\Delta t}-h_{t}\right)^{2} d \mathcal{H}^{1}
\end{aligned}
$$


Then we estimate as usual

$$
\begin{aligned}
\left\|\partial_{\sigma \sigma}\left(Q_{t+\Delta t}-Q_{t}\right)\right\|_{L^{2}} & \leq C\left\|\partial_{\sigma \sigma}\left(Q_{t+\Delta t}-Q_{t}\right)\right\|_{C^{0, \alpha}} \leq C\left\|h_{t+\Delta t}-h_{t}\right\|_{C^{3, \alpha}} \\
& \leq C\left\|\partial_{\sigma \sigma \sigma \sigma}\left(h_{t+\Delta t}-h_{t}\right)\right\|_{L^{2}}^{\vartheta}\left\|h_{t+\Delta t}-h_{t}\right\|_{L^{2}}^{1-\vartheta}+C\left\|h_{t+\Delta t}-h_{t}\right\|_{L^{2}} .
\end{aligned}
$$

Thus, from (3.45) one gets

$$
\int_{\partial G}\left(\dot{h}_{t+\Delta t}-\dot{h}_{t}\right)^{2} d \mathcal{H}^{1} \leq C \int_{\partial G}\left|\partial_{\sigma \sigma \sigma \sigma}\left(h_{t+\Delta t}-h_{t}\right)\right|^{2} d \mathcal{H}^{1}+C \int_{\partial G}\left(h_{t+\Delta t}-h_{t}\right)^{2} d \mathcal{H}^{1} .
$$

Dividing this inequality by $(\Delta t)^{2}$ and recalling what was proved in Step 3 we conclude that for every interval $J \subset \subset(0, T)$

$$
\sup _{t \in J}\left\|\partial_{t t} h_{t}\right\|_{L^{2}(\partial G)}^{2}<\infty .
$$

Similarly, differentiating $k$ times the equation (3.38) and arguing as before we conclude that indeed for every integer $k$ and for every interval $J \subset \subset(0, T)$

$$
\sup _{t \in J}\left\|\partial_{t t}\left(\partial_{\sigma}^{k} h_{t}\right)\right\|_{L^{2}(\partial G)}^{2}<\infty .
$$

Then we have that $h \in W_{l o c}^{2, \infty}\left(0, T ; C^{\infty}(\partial G)\right)$. Finally, differentiating (3.38) with respect to $t$ and repeating the same argument as before we end up by proving that $h \in W_{l o c}^{k, \infty}\left(0, T ; C^{\infty}(\partial G)\right)$ for every integer $k \geq 2$. This concludes the proof.

\section{Asymptotic Stability}

In this section we address the long-time behavior of the flow for a special class of initial data.

To this aim, we start by noticing that if $G$ is stationary, then a standard bootstrap argument shows that in fact $G$ is of class $C^{\infty}$. Moreover, by the results in 32 . $G$ turns out to be analytic. Recall also that the definition of stationary set is weaker than the notion of criticality, where one requires the first variation to be constant on the whole $\partial G$ (see Remark 2.5).

However, the above definition fits better in our framework, since during the evolution there is no mass transfer from one Jordan component to the other. More precisely, denoting as before by $F_{t, i}$ the bounded open set enclosed by the $i$-th connected component $\Gamma_{F_{t}, i}$ of $\partial F_{t}$, one has that the area $\left|F_{t, i}\right|$ is preserved during the flow. Indeed, one has

$$
\frac{d}{d s}\left|F_{t+s, i}\right|_{\left.\right|_{s=0}}=\int_{\Gamma_{F_{t}, i}} V_{t} d \mathcal{H}^{1}=\int_{\Gamma_{F_{t}, i}} \partial_{\sigma \sigma} R_{t} d \mathcal{H}^{1}=0 .
$$

We are now ready to state the main result of this section.

Theorem 4.1. Let $G \subset \subset \Omega$ be a regular strictly stable stationary set in the sense of Definition [2.6 and fix $M>0, \alpha \in(0,1)$. There exists $\delta_{0}>0$ with the following property: Let $F_{0} \in \mathfrak{h}_{M}^{2, \alpha}(\partial G)$ be such that

$$
\left|F_{0} \Delta G\right|<\delta_{0}, \quad \text { and } \quad \int_{\partial F_{0}}\left(\partial_{\sigma} R_{0}\right)^{2} d \mathcal{H}^{1}<\delta_{0},
$$

where $R_{0}:=g\left(\nu_{F_{0}}\right) k_{F_{0}}-Q\left(E\left(u_{F_{0}}\right)\right)$ on $\partial F_{0}$. Then the unique solution $\left(F_{t}\right)_{t>0}$ of the flow (3.1) with intial datum $F_{0}$ is defined for all times $t>0$.

Moreover $F_{t} \rightarrow F_{\infty} H^{3}$-exponentially fast, where $F_{\infty}$ is the unique stationary set in $\mathfrak{h}_{\sigma_{1}}^{2, \alpha}(\partial G)$ (see Proposition 2.9) such that $\left|F_{\infty, i}\right|=\left|F_{0, i}\right|$ for $i=1, \ldots, m$. In particular, if $\left|F_{0, i}\right|=\left|G_{i}\right|$ for $i=1, \ldots, m$, then $F_{t} \rightarrow G H^{3}$-exponentially fast. 
Here $\left(F_{\infty, i}\right)_{i=1, \ldots, m}$ and $\left(F_{0, i}\right)_{i=1, \ldots, m}$ denote the open sets enclosed by the connected components $\left(\Gamma_{F_{\infty}, i}\right)_{i=1, \ldots, m}$ of $\partial F_{\infty}$ and $\left(\Gamma_{F_{0}, i}\right)_{i=1, \ldots, m}$ of $\partial F_{0}$, respectively, numbered according to (2.19).

Remark 4.2. In the previous statement by $H^{3}$ exponential convergence of $F_{t}$ to $F_{\infty}$ we mean precisely the following: writing $\partial F_{t}:=\left\{x+\tilde{h}_{t}(x) \nu_{F_{\infty}}(x): x \in \partial F_{\infty}\right\}$, we have

$$
\left\|\tilde{h}_{t}\right\|_{H^{3}\left(\partial F_{\infty}\right)} \leq C e^{-c t} \text {. }
$$

for suitable constants $C, c>0$.

For an example of strictly stable set $G$ to which Theorem 4.1 applies we refer to 14 .

In order to proof the theorem, we need the following preliminary energy identities.

Proposition 4.3. Let $\left(F_{t}\right)_{t \in(0, T)}$ solve (3.1). Then we have:

$$
\frac{d}{d t} J\left(F_{t}\right)=-\int_{\partial F_{t}}\left(\partial_{\sigma} R_{t}\right)^{2} d \mathcal{H}^{1}
$$

and

$$
\frac{d}{d t}\left(\frac{1}{2} \int_{\partial F_{t}}\left(\partial_{\sigma} R_{t}\right)^{2} d \mathcal{H}^{1}\right)=-\partial^{2} J\left[\partial_{\sigma \sigma} R_{t}\right]-\frac{1}{2} \int_{\partial F_{t}} k_{t}\left(\partial_{\sigma} R_{t}\right)^{2} \partial_{\sigma \sigma} R_{t} d \mathcal{H}^{1}
$$

Proof. The first identity follows immediately recalling that $R_{t}=g\left(\nu_{t}\right) k_{t}-Q\left(E\left(u_{t}\right)\right)$ is the first variation of the energy at $J\left(F_{t}\right)$, and thus

$$
\frac{d}{d t} J\left(F_{t}\right)=\int_{\partial F_{t}} R_{t}\left(X_{t} \cdot \nu_{t}\right) d \mathcal{H}^{1}=\int_{\partial F_{t}} R_{t} \partial_{\sigma \sigma} R_{t} d \mathcal{H}^{1}=-\int_{\partial F_{t}}\left(\partial_{\sigma} R_{t}\right)^{2} d \mathcal{H}^{1}
$$

For the second identity we note that the calculations leading to (3.15) still apply with $f_{t} \circ \pi$ replaced by $-Q\left(E\left(u_{t}\right)\right)$ on $\partial F_{t}$. Hence we have

$$
\begin{aligned}
\frac{d}{d t}\left(\frac{1}{2} \int_{\partial F_{t}}\left(\partial_{\sigma} R_{t}\right)^{2} d \mathcal{H}^{1}\right)= & -\int_{\partial F_{t}} g\left(\nu_{t}\right)\left(\partial_{\sigma \sigma \sigma} R_{t}\right)^{2} d \mathcal{H}^{1}+\int_{\partial F_{t}} \partial_{\sigma \sigma} R_{t} \frac{\partial}{\partial t}\left(Q(E(u(\cdot, t))) d \mathcal{H}^{1}\right. \\
& +\int_{\partial F_{t}} g\left(\nu_{t}\right) k_{t}^{2}\left(\partial_{\sigma \sigma} R_{t}\right)^{2} d \mathcal{H}^{1}+\int_{\partial F_{t}} \partial_{\nu_{t}}\left(Q\left(E\left(u_{t}\right)\right)\right)\left(\partial_{\sigma \sigma} R_{t}\right)^{2} d \mathcal{H}^{1} \\
& -\frac{1}{2} \int_{\partial F_{t}} k_{t}\left(\partial_{\sigma} R_{t}\right)^{2} \partial_{\sigma \sigma} R_{t} d \mathcal{H}^{1} .
\end{aligned}
$$

In order to conclude, we need to show that

$$
\int_{\partial F_{t}} \partial_{\sigma \sigma} R_{t} \frac{\partial}{\partial t}\left(Q(E(u(\cdot, t))) d \mathcal{H}^{1}=2 \int_{\Omega \backslash F_{t}} Q\left(E\left(\dot{u}_{t}\right)\right) d x\right.
$$

to recognize the quadratic form $-\partial^{2} J\left[\partial_{\sigma \sigma} R_{t}\right]$ in the four first terms of (4.2). To this aim, observe that since $\mathbb{C} E\left(u_{t}\right)\left[\nu_{t}\right]=0$ on $\partial F_{t}$, we have

$$
\begin{aligned}
\int_{\partial F_{t}} \partial_{\sigma \sigma} R_{t} & \frac{\partial}{\partial t}\left(Q(E(u(\cdot, t))) d \mathcal{H}^{1}=\int_{\partial F_{t}} R_{\sigma \sigma} \mathbb{C} E\left(u_{t}\right): E\left(\dot{u}_{t}\right) d \mathcal{H}^{1}=\int_{\partial F_{t}} R_{\sigma \sigma} \mathbb{C} E\left(u_{t}\right): D \dot{u}_{t} d \mathcal{H}^{1}\right. \\
& =\int_{\partial F_{t}} \partial_{\sigma \sigma} R_{t} \mathbb{C} E\left(u_{t}\right): D_{\tau} \dot{u}_{t} d \mathcal{H}^{1}=-\int_{\partial F_{t}} \operatorname{div}_{\tau}\left(\partial_{\sigma \sigma} R_{t} \mathbb{C} E\left(u_{t}\right)\right) \cdot \dot{u}_{t} d \mathcal{H}^{1} \\
& =2 \int_{\Omega \backslash F_{t}} Q\left(E\left(\dot{u}_{t}\right)\right) d x
\end{aligned}
$$


where the last equality follows by choosing $\varphi=\dot{u}_{t}$ as a test function in the equation (3.22).

Proof of Theorem 4.1. Throughout the proof, $C$ will denote a constant depending only on the $C^{2, \alpha}$-bounds on the boundary of the set $G$. Here we always assume that $\alpha<1 / 2$ and the value of $C$ may change from line to line. For any set $F \in \mathfrak{h}_{M}^{2, \alpha}(G)$ consider

$$
D(F):=\int_{F \Delta G} \operatorname{dist}(x, \partial G) d x
$$

and note that

$$
|F \Delta G| \leq C\left\|h_{F}\right\|_{L^{2}(\partial G)} \leq C \sqrt{D(F)}
$$

for constants depending only on $G$. Recall that $h_{F}$ is the function such that

$$
\partial F=\left\{x+h_{F}(x) \nu_{G}(x): x \in \partial G\right\} .
$$

For every $\varepsilon_{1}>0$ sufficiently small, there exists $\delta_{1} \in(0,1)$ so small that for any set $F \in$ $\mathfrak{h}_{M}^{2, \alpha}(G)$ the following implications hold true:

$$
F \in \mathfrak{h}_{M}^{2, \alpha}(G) \text { and } D(F) \leq \delta_{1} \Longrightarrow\left\|h_{F}\right\|_{C^{1, \alpha}(\partial G)} \leq \frac{\varepsilon_{1}}{2},
$$

and

$$
\left\|h_{F}\right\|_{C^{1, \alpha}(\partial G)} \leq \varepsilon_{1} \text { and } \int_{\partial F}\left(\partial_{\sigma} R_{F}\right)^{2} d \mathcal{H}^{1} \leq 1 \Longrightarrow\left\|h_{F}\right\|_{C^{2, \alpha}(\partial G)} \leq \omega\left(\varepsilon_{1}\right) \leq \sigma_{1} \wedge M,
$$

where $\omega$ is a positive non-decreasing function such that $\omega\left(\varepsilon_{1}\right) \rightarrow 0$ as $\varepsilon_{1} \rightarrow 0^{+}$, and $\sigma_{1}$ is the constant provided by Proposition 2.9, Here $R_{F}$ stands, as usual, for $g\left(\nu_{F}\right) k_{F}-Q\left(E\left(u_{F}\right)\right)$ on $\partial F$.

Fix $\varepsilon_{1}, \delta_{1} \in(0,1)$ satisfying (4.4) and (4.5) and choose an initial set $F_{0} \in \mathfrak{h}_{M}^{2, \alpha}(G)$ such that

$$
D\left(F_{0}\right) \leq \delta_{0} \quad \text { and } \quad \int_{\partial F_{0}}\left(\partial_{\sigma} R_{0}\right)^{2} d \mathcal{H}^{1} d x \leq \delta_{0},
$$

where the choice of $\delta_{0}<\delta_{1}$ will be made later. Here, we denote $R_{0}$ instead of $R_{F_{0}}$.

Let $\left(F_{t}\right)_{t \in\left(0, T\left(F_{0}\right)\right)}$ the unique classical solution of the flow (3.1) provided by Theorem 3.2. Here $T(F) \in(0,+\infty]$ stands for the maximal time of existence of the classical solution starting from $F$. By the same theorem, there exists $\delta>0$ and $T_{0}>0$ such that

$$
T(F) \geq T_{0} \quad \text { for all } F \subset \subset \Omega \text { s.t. }\left\|h_{F}\right\|_{L^{2}(\partial G)} \leq \delta \text { and }\left\|h_{F}\right\|_{H^{3}(\partial G)} \leq 1 .
$$

Without loss of generality, in what follows we may also assume $\delta_{1}$ to be so small that $D(F) \leq$ $\delta_{1}$ implies $\left\|h_{F}\right\|_{L^{2}(\partial G)} \leq \delta$, with $\delta$ as in (4.7).

We now split the rest of the proof into two steps.

Step 1.(Stopping-time) Let $\bar{t} \leq T\left(F_{0}\right)$ be the maximal time such that

$$
\left\|h_{t}\right\|_{C^{1, \alpha}(\partial G)}<\varepsilon_{1} \text { and } \int_{\partial F_{t}}\left(\partial_{\sigma} R_{t}\right)^{2} d \mathcal{H}^{1}<2 \delta_{0} \text {. for all } t \in(0, \bar{t}),
$$

Note that such a maximal time is well defined in view of (4.4) and (4.6). We claim that by taking $\varepsilon_{1}$ and $\delta_{0}$ smaller if needed, we have $\bar{t}=T\left(F_{0}\right)$. To this aim, assume by contradiction that $\bar{t}<T\left(F_{0}\right)$. Then,

$$
\left\|h_{\bar{t}}\right\|_{C^{1, \alpha}(\partial G)}=\varepsilon_{1} \quad \text { or } \quad \int_{\partial F_{\bar{t}}}\left(\partial_{\sigma} R_{\bar{t}}\right)^{2} d \mathcal{H}^{1}=2 \delta_{0}
$$

We split the proof into steps, according to the two alternatives above. 
Step 1-(a). Assume that

$$
\int_{\partial F_{\bar{t}}}\left(\partial_{\sigma} R_{\bar{t}}\right)^{2} d \mathcal{H}^{1}=2 \delta_{0} .
$$

Since (4.5) holds for $h_{t}$ for every $t \in(0, \bar{t})$ then by Lemma 2.7 (and the fact that $\sigma_{1}<\sigma_{0}$ ) we get

$$
J\left(F_{t}\right)\left[\partial_{\sigma \sigma} R_{t}\right] \geq \frac{m_{0}}{2}\left\|\partial_{\sigma \sigma} R_{t}\right\|_{H^{1}\left(\partial F_{t}\right)}^{2} .
$$

Therefore by Proposition 4.3 we get

$$
\begin{aligned}
\frac{d}{d t}\left(\frac{1}{2} \int_{\partial F_{t}}\left(\partial_{\sigma} R_{t}\right)^{2}\right. & \left.d \mathcal{H}^{1}\right)=-J\left(F_{t}\right)\left[\partial_{\sigma \sigma} R_{t}\right]-\frac{1}{2} \int_{\partial F_{t}} k_{t}\left(\partial_{\sigma} R_{t}\right)^{2} \partial_{\sigma \sigma} R_{t} d \mathcal{H}^{1} \\
& \leq-\frac{m_{0}}{2}\left\|\partial_{\sigma \sigma} R_{t}\right\|_{H^{1}\left(\partial F_{t}\right)}^{2}+C \int_{\partial F_{t}}\left(\partial_{\sigma} R_{t}\right)^{2}\left|\partial_{\sigma \sigma} R_{t}\right| d \mathcal{H}^{1} \\
& \leq-\frac{m_{0}}{2} \int_{\partial F_{t}}\left(\partial_{\sigma \sigma \sigma} R_{t}\right)^{2} d \mathcal{H}^{1}+C \int_{\partial F_{t}}\left|\partial_{\sigma} R_{t}\right|^{3}+\left|\partial_{\sigma \sigma} R_{t}\right|^{3} d \mathcal{H}^{1}
\end{aligned}
$$

In turn, by Proposition 6.1 and the Poincaré Inequality we get

$$
\begin{aligned}
\left\|\partial_{\sigma} R_{t}\right\|_{L^{3}\left(\partial F_{t}\right)}^{3} & \leq C\left\|\partial_{\sigma \sigma \sigma} R_{t}\right\|_{L^{2}\left(\partial F_{t}\right)}^{\frac{1}{4}}\left\|\partial_{\sigma} R_{t}\right\|_{L^{2}\left(\partial F_{t}\right)}^{\frac{11}{4}} \\
& \leq C\left\|\partial_{\sigma \sigma \sigma} R_{t}\right\|_{L^{2}\left(\partial F_{t}\right)}^{2}\left\|\partial_{\sigma} R_{t}\right\|_{L^{2}\left(\partial F_{t}\right)} \\
& \leq C \sqrt{\delta_{0}}\left\|\partial_{\sigma \sigma \sigma} R_{t}\right\|_{L^{2}\left(\partial F_{t}\right)}^{2},
\end{aligned}
$$

where the last inequality follows from (4.8). Similarly we get

$$
\begin{aligned}
\left\|\partial_{\sigma \sigma} R_{t}\right\|_{L^{3}\left(\partial F_{t}\right)}^{3} & \leq C\left\|\partial_{\sigma \sigma \sigma} R_{t}\right\|_{L^{2}\left(\partial F_{t}\right)}^{\frac{7}{4}}\left\|\partial_{\sigma} R_{t}\right\|_{L^{2}\left(\partial F_{t}\right)}^{\frac{5}{4}} \\
& \leq C \sqrt{\delta_{0}}\left\|\partial_{\sigma \sigma \sigma} R_{t}\right\|_{L^{2}\left(\partial F_{t}\right)}^{2} .
\end{aligned}
$$

Therefore, choosing $\delta_{0}$ small enough, we deduce from (4.10) that

$$
\begin{aligned}
\frac{d}{d t}\left(\frac{1}{2} \int_{\partial F_{t}}\left(\partial_{\sigma} R_{t}\right)^{2} d \mathcal{H}^{1}\right) & \leq\left(-\frac{m_{0}}{2}+C \sqrt{\delta_{0}}\right) \int_{\partial F_{t}}\left(\partial_{\sigma \sigma \sigma} R_{t}\right)^{2} d \mathcal{H}^{1} \\
& \leq-\frac{m_{0}}{4} \int_{\partial F_{t}}\left(\partial_{\sigma \sigma \sigma} R_{t}\right)^{2} d \mathcal{H}^{1} \\
& \leq-m_{1} \int_{\partial F_{t}}\left(\partial_{\sigma} R_{t}\right)^{2} d \mathcal{H}^{1}
\end{aligned}
$$

for all $t \leq \bar{t}$ and for some $m_{1}>0$. Note that the last inequality above follows from the Poincaré Inequality. Integrating the above differential inequality implies

$$
\int_{\partial F_{t}}\left(\partial_{\sigma} R_{t}\right)^{2} d \mathcal{H}^{1} \leq e^{-m_{1} t} \int_{\partial F_{0}}\left(\partial_{\sigma} R_{0}\right)^{2} d \mathcal{H}^{1} \leq \delta_{0} e^{-m_{1} t}
$$

for every $t \leq \bar{t}$. This contradicts (4.9).

Step 1-(b). Assume now that

$$
\left\|h_{\bar{t}}\right\|_{C^{1, \alpha}(\partial F)}=\varepsilon_{1} .
$$


By (3.8) we have that

$$
\frac{d}{d t} D\left(F_{t}\right) \leq P\left(F_{t}\right)^{\frac{1}{2}}\left(\int_{\partial F_{t}}\left(\partial_{\sigma} R_{t}\right)^{2} d \mathcal{H}^{1}\right)^{\frac{1}{2}},
$$

where $D\left(F_{t}\right)$ is defined in (4.3). Therefore we may use (4.11) to estimate

$$
\frac{d}{d t} D\left(F_{t}\right) \leq C \sqrt{\delta_{0} e^{-m_{1} t}}
$$

for every $t \leq \bar{t}$. This implies

$$
D\left(F_{t}\right) \leq D\left(F_{0}\right)+C \sqrt{\delta_{0}} \leq C \sqrt{\delta_{0}}
$$

for every $t \leq \bar{t}$. We may choose $\delta_{0}$ so small enough the above estimate implies $D\left(F_{t}\right) \leq \delta_{1}$ and, in turn, by (4.4), $\left\|h_{t}\right\|_{C^{1, \alpha}(\partial F)} \leq \frac{\varepsilon_{1}}{2}$ for every $t \leq \bar{t}$. This contradicts (4.12).

Step 2.(Global-in-time existence and convergence) By the previous step we have that as long as the flow is defined, i.e., over $\left(0, T\left(F_{0}\right)\right)$, the estimates (4.8) hold. In turn, by taking $\varepsilon_{1}$ (and $\left.\delta_{1}, \delta_{0}\right)$ smaller if needed, we have $\left\|h_{t}\right\|_{L^{2}(\partial G)} \leq \delta$ and $\left\|h_{t}\right\|_{H^{3}(\partial G)} \leq 1$ for all $t \in\left(0, T\left(F_{0}\right)\right)$. By (4.7) and a standard continuation argument, we deduce that $\left(F_{t}\right)_{t}$ is defined for all times, i.e., $T\left(F_{0}\right)=\infty$.

From (4.11) we also deduce that

$$
\int_{\partial F_{t}}\left(\partial_{\sigma} R_{t}\right)^{2} d \mathcal{H}^{1} \leq e^{-m_{1} t} \int_{\partial F_{0}}\left(\partial_{\sigma} R_{0}\right)^{2} d \mathcal{H}^{1} \leq \delta_{0} e^{-m_{1} t}
$$

for all $t>0$, and in turn, by (4.5) we have

$$
\left\|h_{t}\right\|_{C^{2, \alpha}(\partial G)} \leq M
$$

for all $t>0$. Therefore, we deduce that there exists $h_{\infty} \in C^{2, \alpha}(\partial G)$ and a sequence $t_{n} \rightarrow+\infty$ such that

$$
h_{t_{n}} \rightarrow h_{\infty} \quad \text { in } C^{2, \beta}(\partial G) \text { for all } \beta<\alpha .
$$

Moreover, by (4.15) we have $\partial_{\sigma} R_{\infty}=0$, and thus, the set $F_{\infty} \in \mathfrak{h}_{M}^{2, \alpha}(G)$ such that

$$
\partial F_{\infty}=\left\{x+h_{\infty}(x) \nu_{G}(x): x \in \partial G\right\}
$$

is stationary. Recall that for every $t \in[0,+\infty],\left(\Gamma_{F_{t}, i}\right)_{i=1, \ldots, m}$ denote the connected components of $\partial F_{t}$, numbered according to (2.19). Denote also as usual by $F_{t, i}$ the bounded open set enclosed by $\Gamma_{F_{t}, i}$. Since $\left|F_{t, i}\right|=\left|F_{i, 0}\right|$ for every $t>0$ by (4.1), taking also into account (4.5) and Proposition 2.9, we deduce that in fact $F_{\infty}$ is the unique stationary set in $\mathfrak{h}_{\sigma_{1}}^{2, \alpha}(\partial G)$ such that $\left|F_{\infty, i}\right|=\left|F_{0, i}\right|$ for $i=1, \ldots, m$.

It remains to show that the whole flow exponentially converges to $F_{\infty}$. To this aim, define

$$
D_{\infty}(E):=\int_{E \Delta F_{\infty}} \operatorname{dist}\left(x, \partial F_{\infty}\right) d x .
$$

The same calculations and arguments leading to (4.13) and (4.14) show that

$$
\frac{d}{d t} D_{\infty}\left(F_{t}\right) \leq P\left(F_{t}\right)^{\frac{1}{2}}\left(\int_{\partial F_{t}}\left(\partial_{\sigma} R_{t}\right)^{2} d \mathcal{H}^{1}\right)^{\frac{1}{2}} \leq C \sqrt{\delta_{0} e^{-m_{1} t}}
$$


for all $t>0$. From this inequality it is easy to deduce that $\lim _{t \rightarrow+\infty} D_{\infty}\left(F_{t}\right)$ exists. Thus, by (4.17), $D_{\infty}\left(F_{t}\right) \rightarrow 0$ as $t \rightarrow+\infty$. In turn, integrating (4.18) and writing $\partial F_{t}=\{x+$ $\left.\tilde{h}_{t}(x) \nu_{F_{\infty}}(x): x \in \partial F_{\infty}\right\}$ we get

$$
\left\|\tilde{h}_{t}\right\|_{L^{2}\left(\partial F_{\infty}\right)}^{2} \leq C D_{\infty}\left(F_{t}\right) \leq \int_{t}^{+\infty} C \sqrt{\delta_{0} e^{-m_{1} s}}, d s \leq C \sqrt{\delta_{0} e^{-m_{1} t}} .
$$

Since $\left(\tilde{h}_{t}\right)_{t>0}$ are bounded in $C^{2, \alpha}(\partial G)$ by (4.16), we obtain by the above estimate together with standard interpolation that also $\left\|\tilde{h}_{t}\right\|_{C^{2, \beta}(\partial G)} \rightarrow 0$ exponentially fast to zero for $\beta<\alpha$. Finally, using also (4.15) and Lemma 3.6 (with $G=F_{\infty}$ ), we deduce that $\left\|\tilde{h}_{t}\right\|_{H^{3}(\partial G)} \rightarrow 0$ exponentially fast.

\section{PERIODIC GRAPHS}

In this section we briefly describe how our main results read in the context of evolving periodic graphs.

In this framework, given a (sufficiently regular) non-negative $\ell$-periodic function $h:[0, \ell] \rightarrow$ $[0,+\infty)$, the free energy associated with it reads

$$
J(h):=\int_{\Omega_{h}} Q\left(E\left(u_{h}\right)\right) d x+\int_{\Gamma_{h}} \varphi\left(\nu_{\Omega_{h}}\right) d \mathcal{H}^{1},
$$

where $x=\left(x_{1}, x_{2}\right) \in \mathbb{R}^{2}, \Gamma_{h}$ denotes the graph of $h, \Omega_{h}$ is the subgraph of $h$, i.e.,

$$
\Omega_{h}:=\left\{\left(x_{1}, x_{2}\right) \in(0, \ell) \times \mathbb{R}: 0<x_{2}<h\left(x_{1}\right)\right\},
$$

and $u_{h}$ is the elastic equilibrium in $\Omega_{h}$, namely the solution of the elliptic system

$$
\left\{\begin{array}{l}
\operatorname{div} \mathbb{C} E\left(u_{h}\right)=0 \text { in } \Omega_{h}, \\
\mathbb{C} E\left(u_{h}\right)\left[\nu_{\Omega_{h}}\right]=0 \text { on } \Gamma_{h}, \\
\nabla u_{h}\left(\cdot, x_{2}\right) \text { is } \ell \text {-periodic, } \\
u\left(x_{1}, 0\right)=e_{0}\left(x_{1}, 0\right),
\end{array}\right.
$$

for a suitable fixed constant $e_{0} \neq 0$. As mentioned already in the introduction, the above energy relates to a variational model for epitaxial growth: The graph $\Gamma_{h}$ describes the (free) profile of the elastic films, which occupies the region $\Omega_{h}$ and is grown on a (rigid) and much thicker substrate, while the mismatch strain constant $e_{0}$ appearing in the Dirichlet condition for $u_{h}$ at the interface $\left\{x_{1}=0\right\}$ between film and substrate measures the mismatch between the characteristic atomic distances in the lattices of the two materials. In this framework, the (local) minimizers of (5.1) under an area constraint on $\Omega_{h}$ describe the equilibrium configurations of epitaxially strained elastic films, see [21, 22, 23, 25] and the reference therein.

In the context of periodic graphs, given an initial $\ell$-periodic profile $\bar{h} \in H^{3}(0, \ell)$ (in short $\left.\bar{h} \in H_{\text {per }}^{3}(0, \ell)\right)$, we look for a solution $\left(h_{t}\right)_{t \in[0, T)}$ of the following problem:

$$
\begin{cases}\frac{1}{J_{t}} \dot{h}_{t}=\left(g\left(\nu_{t}\right) k_{t}+Q\left(E\left(u_{t}\right)\right)\right)_{\sigma \sigma} & \text { on } \Gamma_{h_{t}} \text { and for all } t \in(0, T), \\ h_{t} \text { is } \ell \text {-periodic } & \text { for all } t \in(0, T), \\ h_{0}=\bar{h} & \end{cases}
$$

where we set $J_{t}:=\sqrt{1+\left|\frac{\partial h_{t}}{\partial x_{1}}\right|^{2}}, u_{t}$ stands for the solution of (15.2), with $\Omega_{h_{t}}$ in place of $\Omega_{h}$, and we wrote $\nu_{t}, k_{t}$ instead of $\nu_{\Omega_{h_{t}}}$ and $k_{\Omega_{h_{t}}}$, respectively. Note that in the first equation 
of (5.3) we have $+Q\left(E\left(u_{t}\right)\right)$ instead of $-Q\left(E\left(u_{t}\right)\right)$. This is due to the fact that in (5.1) the vector $\nu_{\Omega_{h}}$ now point outwards with respect to the elastic body.

Although the setting is slightly different from that of the previous sections, the short-time existence and regularity theory of Section 3 clearly extends also to the present situation, with the same arguments. In this way we improve upon the results of [22], showing that there is no need of a curvature regularization in the case where the anisotropy $\varphi$ is convex and satisfies the ellipticity condition (2.8). Also the stabilty analysis of Section 4 goes through without changes, thus showing that strictly stable stationary $\ell$-periodic configurations are $H^{3}$-exponentially stable (in the sense made precise by Remark 4.2).

In the case of flat configurations, that is, of constant profiles $h \equiv a$ for some $a>0$, and when $Q$ is of the form

$$
Q(E):=\mu|E|^{2}+\frac{\lambda}{2}(\text { trace } E)^{2}
$$

for some constants $\mu>0$ and $\lambda>-\mu$ (the so called Lamé coefficients), the relation between the $a, \mu, \lambda, \ell$, and $e_{0}$ (see (5.2)) that guarantees the strict stability of flat configuration $h \equiv a$ with respect to $\ell$-periodic perturbations is analytically determined. For the reader's convenience, we recall the results. Consider the Grinfeld function $K$ defined by

$$
K(s):=\max _{n \in \mathbb{N}} \frac{1}{n} H(n s), \quad s \geq 0,
$$

where

$$
H(s):=\frac{y+\left(3-4 \nu_{p}\right) \sinh s \cosh s}{4\left(1-\nu_{p}\right)^{2}+s^{2}+\left(3-4 \nu_{p}\right) \sinh ^{2} s},
$$

and $\nu_{p}$ is the Poisson modulus of the elastic material, i.e., $\nu_{p}:=\frac{\lambda}{2(\lambda+\mu)}$.

It turns out that $K$ is strictly increasing and continuous, $K(s) \leq C s$, and $\lim _{s \rightarrow+\infty} K(s)=1$, for some positive constant $C$, see [25. Corollary 5.3]. Set $\mathbf{e}_{1}:=(1,0)$ and $\mathbf{e}_{2}:=(0,1)$. Combining [25, Theorem 2.9] and [8, Theorem 2.8] with the results of the previous section, we obtain the following theorem.

Theorem 5.1. Let $a_{\text {stable }}:(0,+\infty) \rightarrow(0,+\infty]$ be defined as $a_{\text {stable }}(\ell):=+\infty$, if $0<\ell \leq$ $\frac{\pi}{4} \frac{(2 \mu+\lambda) \partial_{\mathbf{e}_{1}} \mathbf{e}_{1} \varphi\left(\mathbf{e}_{2}\right)}{e_{0}^{2} \mu(\mu+\lambda)}$, and as the solution of

$$
K\left(\frac{2 \pi a_{\text {stable }}(\ell)}{\ell}\right)=\frac{\pi}{4} \frac{(2 \mu+\lambda) \partial_{\mathbf{e}_{\mathbf{1}} \mathbf{e}_{\mathbf{1}}} \varphi\left(\mathbf{e}_{\mathbf{2}}\right)}{e_{0}^{2} \mu(\mu+\lambda)} \frac{1}{\ell},
$$

otherwise. Then $h \equiv a$ is an $\ell$-periodic strictly stable stationary configuration for (5.1) if and only if $0<a<a_{\text {stable }}(\ell)$. In particular, for all $a \in\left(0, a_{\text {stable }}(\ell)\right)$ there exists $\delta>0$ such that if $\|\bar{h}-a\|_{H_{\mathrm{per}}^{3}(0, \ell)} \leq \delta$ and $\left|\Omega_{\bar{h}}\right|=a \ell$, then the unique solution $\left(h_{t}\right)_{t}$ of (5.3) is defined for all times and satisfies

$$
\left\|h_{t}-a\right\|_{H_{\mathrm{per}}^{3}(0, \ell)} \leq C e^{-c t} \quad \text { for all } t>0,
$$

for suitable constants $C, c>0$.

\section{Appendix}

Let $s \in(0,1)$ and $p \geq 1$. We recall that for a function $f: \mathbb{S}^{1} \rightarrow \mathbb{R}$ the Gagliardo seminorm $[f]_{s, p}$ is defined as

$$
[f]_{s, p}^{p}:=\int_{\mathbb{S}^{1}} \int_{\mathbb{S}^{1}} \frac{|f(x)-f(y)|^{p}}{|x-y|^{1+s p}} d x d y
$$


If $s>0$ and $\ell$ is the integer part of $s$, the Sobolev space $W^{s, p}\left(\mathbb{S}^{1}\right)$ is the space of all functions $f$ in $W^{\ell, p}\left(\mathbb{S}^{1}\right)$ such that $\left[\partial^{\ell} f\right]_{s-\ell, p}$ is finite, endowed with the norm $\|f\|_{W^{s, p}\left(\mathbb{S}^{1}\right)}^{p}:=\|f\|_{W^{\ell, p}\left(\mathbb{S}^{1}\right)}^{p}+$ $\left[\partial_{\sigma}^{\ell} f\right]_{s-\ell, p}^{p}$. Here we used the convention $W^{0, p}=L^{p}$ and $\left[\partial_{\sigma}^{\ell} f\right]_{s-\ell, p}^{p}=\left\|\partial_{\sigma}^{\ell} f\right\|_{L^{p}(\Gamma)}$. We recall also that for $p=2$ the seminorm $\left[\partial_{\sigma}^{\ell} f\right]_{s-\ell, p}^{p}$ is equivalent to

$$
\left(\sum_{k \in \mathbb{Z}} k^{2 s} a_{k}(f)^{2}\right)^{\frac{1}{2}}
$$

where $\left\{a_{k}(f)\right\}$ is the sequence of the Fourier coefficients of $f$ with respect to the orthornormal basis $\left\{(2 \pi)^{\frac{1}{2}} \mathrm{e}^{-i k z}\right\}_{k \in \mathbb{Z}}$. These definitions extend in the obvious way to the case where $\mathbb{S}^{1}$ is replaced by any regular Jordan curve $\Gamma$.

We prove the following interpolation inequality for curves. Note that in the statement we are using and $W^{t, 2}=H^{t}$ for all $t>0$.

Proposition 6.1. Let $\Gamma$ be a regular Jordan curve. Let $m \geq 1$ be an integer, $0 \leq s<m$ and $p \in[2,+\infty)$ such that $s+1 / 2-1 / p<m$. There exists a constant $C>0$, depending only on $m, s, p$ and on the length of $\Gamma$ such that for every $f \in H^{m}(\Gamma)$

$$
\|f\|_{W^{s, p}(\Gamma)} \leq C\left(\left\|\partial_{\sigma}^{m} f\right\|_{L^{2}(\Gamma)}^{\theta}\|f\|_{L^{2}(\Gamma)}^{1-\theta}+\|f\|_{L^{2}(\Gamma)}\right),
$$

where

$$
\theta=\frac{s+1 / 2-1 / p}{m}
$$

If $s$ is a positive integer, then

$$
\left\|\partial_{\sigma}^{s} f\right\|_{L^{p}(\Gamma)} \leq C\left\|\partial_{\sigma}^{m} f\right\|_{L^{2}(\Gamma)}^{\theta}\|f\|_{L^{2}(\Gamma)}^{1-\theta}
$$

with $\theta$ as before. The same inequality also holds if $s=0$, provided that $f$ has zero average.

Finally, if $0<\alpha<\frac{1}{2}$, there exists $\theta^{\prime}$, depending only on $m$ and $\alpha$, such that for every $f \in H^{m}(\Gamma)$

$$
\|f\|_{C^{m-1, \alpha}(\Gamma)} \leq C\left(\left\|\partial_{\sigma}^{m} f\right\|_{L^{2}(\Gamma)}^{\theta^{\prime}}\|f\|_{L^{2}(\Gamma)}^{1-\theta^{\prime}}+\|f\|_{L^{2}(\Gamma)}\right) .
$$

Proof. It is enough to prove the statement for $\Gamma=\mathbb{S}^{1}$. The general case will follow by parametrizing $\Gamma$ by the arclength and by rescaling. Let $t=s+\frac{1}{2}-\frac{1}{p}$. Observe that

$$
\left(\sum_{k \in \mathbb{Z}}^{\infty} k^{2 t} a_{k}(f)^{2}\right)^{\frac{1}{2}} \leq\left(\sum_{k \in \mathbb{Z}} k^{2 m} a_{k}(f)^{2}\right)^{\frac{\theta}{2}}\left(\sum_{k \in \mathbb{Z}} a_{k}(f)^{2}\right)^{\frac{1-\theta}{2}},
$$

and thus (6.1) follows with $\|f\|_{W^{s, p}\left(\mathbb{S}^{1}\right)}$ replaced by $\|f\|_{W^{t, 2}\left(\mathbb{S}^{1}\right)}$. The general case follows recalling that $W^{t, 2}\left(\mathbb{S}^{1}\right)$ is continuously embedded in $W^{s, p}\left(\mathbb{S}^{1}\right)$, since $t=s+\frac{1}{2}-\frac{1}{p}$ (see [28, Th. 1.4.4.1]). Observe that it is enough to prove (6.2) for functions $f$ with zero average also when $s$ is a positive integer. On the other hand if $f$ has zero average, (6.2) follows from (6.4) and the aforementioned Sobolev Embedding after observing that the $W^{s, p}$-norm of $f$ is equivalent to the $L^{p}$-norm of $\partial_{\sigma}^{s} f$.

Finally, to prove (6.3) it is enough to assume $m=1$ and then to argue by induction with respect to $m$. To this aim, we observe that for every $z, w \in \mathbb{S}^{1}$

$$
|f(z)-f(w)| \leq c|z-w|^{\frac{1}{2}}\left\|\partial_{\sigma} f\right\|_{L^{2}}
$$


for some universal constant $c$. Then if $0<\alpha<\frac{1}{2}$

$$
|f(z)-f(w)| \leq|f(z)-f(w)|^{2 \alpha}|f(z)-f(w)|^{1-2 \alpha} \leq c|z-w|^{\alpha}\left\|\partial_{\sigma} f\right\|_{L^{2}}^{2 \alpha}\|f\|_{L^{\infty}}^{1-2 \alpha} .
$$

The conclusion follows by estimating $\|f\|_{L^{\infty}}$ by (6.1) , with $m=1$.

\section{REFERENCES}

[1] Acerbi E.; Fusco N.; Julin V.; Morini M., Nonlinear stability results for the modified Mullins-Sekerka and the surface diffusion flow. Preprint 2016. To appear on J. Differential. Geom.

[2] Acerbi E.; Fusco N.; Morini M., Minimality via second variation for a nonlocal isoperimetric problem, Comm. Math. Phys. 322 (2013), 515-557.

[3] Agmon S.; Douglis A.; Nirenberg L., Estimates near the boundary for solutions of elliptic partial differential equations satisfying general boundary conditions. II, Comm. Pure Appl. Math. 17 (1964), $35-92$.

[4] Ambrosetti A.; Prodi G., A primer of nonlinear analysis., Cambridge Studies in Advanced Mathematics, 34. Cambridge University Press, Cambridge, 1995.

[5] Angenent S.; Gurtin M.E., Multiphase thermomechanics with interfacial structure. II. Evolution of an isothermal interface. Arch. Rational Mech. Anal. 108 (1989), 323-391.

[6] Bella P.; Goldman M.; Zwicknagl B., Study of island formation in epitaxially strained films on unbounded domains. Arch. Ration. Mech. Anal. 218 (2015), 163-217.

[7] Bellettini G.; Mantegazza C.; Novaga M., Singular perturbations of mean curvature flow. J. Differential Geom. 75 (2007), 403-431.

[8] Bonacini M., Epitaxially strained elastic films: the case of anisotropic surface energies. ESAIM Control Optim. Calc. Var. 19 (2013), 167-189.

[9] Bonacini M., Stability of equilibrium configurations for elastic films in two and three dimensions. Adv. Calc. Var. 8 (2015), 117-153.

[10] Bonnetier E.; Chambolle A., Computing the equilibrium configuration of epitaxially strained crystalline films. SIAM J. Appl. Math. 62 (2002), 1093-1121.

[11] Braides A.; Chambolle A.; Solci M., A relaxation result for energies defined on pairs set-function and applications. ESAIM Control Optim. Calc. Var. 13 (2007), 717-734.

[12] Burger M.; Hausser H.; Stöcker C.; Voigt A., A level set approach to anisotropic flows with curvature regularization. J. Comput. Phys. 225 (2007), 183-205.

[13] Cahn J. W.; Taylor J. E., Overview N0-113 - Surface motion by surface-diffusion. Acta Metallurgica et Materialia, 42 (1994), 1045-1063.

[14] Capriani G.M.; Julin V.; Pisante G., A quantitative second order minimality criterion for cavities in elastic bodies. SIAM J. Math. Anal. 45 (2013), 1952-1991.

[15] Chambolle A.; Solci M., Interaction of a bulk and a surface energy with a geometrical constraint. SIAM J. Math. Anal. 39 (2007), 77-102.

[16] Chen X., The Hele-Shaw problem and area-preserving curve-shortening motions. Arch. Rational Mech. Anal. 123 (1993), 117-151.

[17] Choksi R.; Sternberg P., On the first and second variations of a nonlocal isoperimetric problem. J. reine angew. Math. 611 (2007), 75-108.

[18] Di Carlo A.; Gurtin M. E.; Podio-Guidugli P., A regularized equation for anisotropic motion-bycurvature. SIAM J. Appl. Math. 52 (1992), 1111-1119.

[19] Elliott C. M.; Garcke H., Existence results for diffusive surface motion laws. Adv. Math. Sci. Appl. 7 (1997), 467-490.

[20] Escher J.; Mayer U. F.; Simonett G., The surface diffusion flow for immersed hypersurfaces. SIAM J. Math. Anal. 29 (1998), 1419-1433.

[21] Fonseca I.; Fusco N.; Leoni G.; Morini M., Equilibrium configurations of epitaxially strained crystalline films: existence and regularity results. Arch. Ration. Mech. Anal. 186 (2007), 477-537.

[22] Fonseca I.; Fusco N.; Leoni G.; Morini M., Motion of elastic thin films by anisotropic surface diffusion with curvature regularization. Arch. Ration. Mech. Anal. 205 (2012), 425-466.

[23] Fonseca I.; Fusco N.; Leoni G.; Morini M., Motion of elastic three-dimensional elastic films by anisotropic surface diffusion with curvature regularization. Anal. PDE 8 (2015), 373-423. 
[24] Fonseca I.; Fusco N.; Leoni G.; Millot V., Material voids in elastic solids with anisotropic surface energies. J. Math. Pures Appl. 96 (2011), 591-639.

[25] Fusco N.; Morini M., Equilibrium configurations of epitaxially strained elastic films: second order minimality conditions and qualitative properties of solutions. Arch. Rational Mech. Anal. 203 (2012), 247-327.

[26] Giga Y; Iто K., On pinching of curves moved by surface diffusion. Commun. Appl. Anal. 2 (1998), 393-405.

[27] Goldman M.; Zwicknagl B., Scaling law and reduced models for epitaxially strained crystalline films. SIAM J. Math. Anal. 46 (2014), 1-24.

[28] Grisvard P., Elliptic problems in nonsmooth domains. Monographs and Studies in Mathematics, 24. Pitman (Advanced Publishing Program), Boston, MA, 1985.

[29] Gurtin M. E.; JABbour M. E., Interface evolution in three dimensions with curvature-dependent energy and surface diffusion: interface-controlled evolution, phase transitions, epitaxial growth of elastic films. Arch. Ration. Mech. Anal. 163 (2002), 171-208.

[30] Gurtin M.; Voorhees P., The continuum mechanics of coherent two-phase elastic solids with mass transport. Proc. R. Soc. Lond. A 440 (1993), 323-343.

[31] Herring C., Some theorems on the free energies of crystal surfaces., Physical Review 82 (1951), 87-93.

[32] Koch H.; Leoni G.; Morini M., On Optimal regularity of Free Boundary Problems and a Conjecture of De Giorgi. Comm. Pure Applied Math. 58 (2005), 1051-1076.

[33] Morini M., Local and global minimality results for an isoperimetric problem with long-range interactions, in 'Free Discontinuity Problems' 153-224, CRM series 19, Ed. Norm., Pisa, 2017.

[34] Mullins W. W., Solid surface morphologies governed by capillarity. In: Metal Surfaces. American society for metals, 1963.

[35] Rätz A.; Ribalta A.; Voigt A., Surface evolution of elastically stressed films under deposition by a diffuse interface model. J. Comp. Phys. 214 (2006), 187-208.

[36] Siegel M.; Miksis M. J.; Voorhees P. W., Evolution of material voids for highly anisotropic surface energy. J. Mech. Phys. Solids 52 (2004), 1319-1353. 